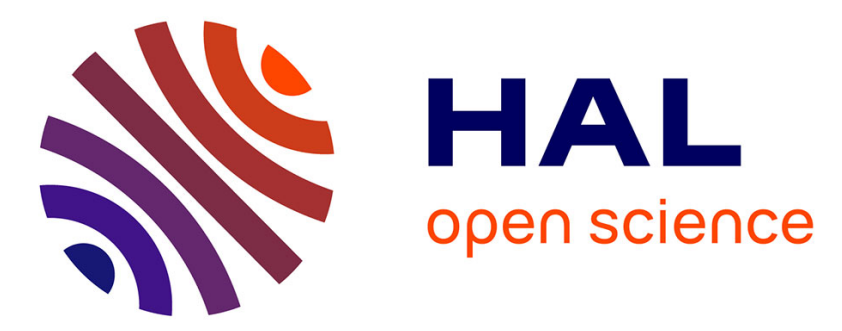

\title{
Modal interactions due to friction in the nonlinear vibration response of the "Harmony" test structure: Experiments and simulations
}

\author{
M Claeys, Jean-Jacques Sinou, J-P Lambelin, R Todeschini
}

\section{To cite this version:}

M Claeys, Jean-Jacques Sinou, J-P Lambelin, R Todeschini. Modal interactions due to friction in the nonlinear vibration response of the "Harmony" test structure: Experiments and simulations. Journal of Sound and Vibration, 2016, 376, pp.131 - 148. 10.1016/j.jsv.2016.04.008 . hal-03255747

\section{HAL Id: hal-03255747 \\ https://hal.science/hal-03255747}

Submitted on 9 Jun 2021

HAL is a multi-disciplinary open access archive for the deposit and dissemination of scientific research documents, whether they are published or not. The documents may come from teaching and research institutions in France or abroad, or from public or private research centers.
L'archive ouverte pluridisciplinaire HAL, est destinée au dépôt et à la diffusion de documents scientifiques de niveau recherche, publiés ou non, émanant des établissements d'enseignement et de recherche français ou étrangers, des laboratoires publics ou privés. 
M. Claeys, J-J. Sinou, J-P. Lambelin and R. Todeschini, Modal interactions due to friction in the nonlinear vibration response of the "Harmony" test structure: experiments and simulations, Journal of Sound and Vibration, 376, 131-148, 2016.

doi:10.1016/j.jsv.2016.04.008

\title{
Modal interactions due to friction in the nonlinear vibra- tion response of the "Harmony" test structure: experi- ments and simulations
}

\author{
M. Claeys ${ }^{a, b}$, J-J. Sinou ${ }^{b, c}$, J-P. Lambelin ${ }^{a}$ and R. Todeschini ${ }^{a}$ \\ ${ }^{a}$ CEA, DAM, CESTA, F-33114 Le Barp, France. \\ ${ }^{b}$ Laboratoire de Tribologie et Dynamique des Systèmes UMR-CNRS 5513, Ecole Centrale de Lyon, \\ 36 avenue Guy de Collongue, 69134 Ecully Cedex, France. \\ ${ }^{c}$ Institut Universitaire de France, 75005 Paris, France.
}

\begin{abstract}
The nonlinear vibration response of an assembly with friction joints -named "Harmony" - is studied both experimentally and numerically. The experimental results exhibit a softening effect and an increase of dissipation with excitation level. Modal interactions due to friction are also evidenced.

The numerical methodology proposed groups together well-known structural dynamic methods, including finite elements, substructuring, Harmonic Balance and continuation methods. On the one hand, the application of this methodology proves its capacity to treat a complex system where several friction movements occur at the same time. On the other hand, the main contribution of this paper is the experimental and numerical study of evidence of modal interactions due to friction. The simulation methodology succeeds in reproducing complex form of dynamic behavior such as these modal interactions.
\end{abstract}




\section{Introduction}

The "Harmony" test structure has been designed to study the effects of friction on the frequency response of an assembly. In a previous work [1], an experimental and numerical study was undertaken on this structure. The experimental frequency response of the assembly to an axial excitation has been studied, highlighting the damping and softening effects due to friction. A methodology which groups together well-known methods of structural dynamics has been proposed to model the structure and simulate its vibration response. Firstly, each component of the assembly and the assembly itself are modeled using finite elements. These models are updated to fit experimental vibration responses at a low excitation level. The assembly model is then reduced using a substructuring algorithm [2]. The nonlinear behavior of the friction joints is introduced at this step: the linear connectors which model joints are replaced by nonlinear elastic Coulomb models. The nonlinear dynamic system obtained is solved using the Harmonic Balance Method [3] coupled with a condensation process [4] and a continuation method. The simulation results obtained reproduce the damping and softening effects due to friction. This initial work has thus proved the capacity of the proposed methodology to simulate the effects of friction in a complex assembly.

In the present paper, the response of the "Harmony" test structure to a transverse excitation is studied. The simulation methodology, based on the Harmonic Balance Method with condensation and predictor-corrector processes, is applied. The first contribution of this new study is to prove the capacity of the methodology to treat a case where diverse friction movements occur at the same time (both translations and rotations occur in the friction movement of the joints). Subsequently, the main contribution of this paper is the experimental and numerical study of modal interactions due to friction. A key element of this study is the use of a 3D scanning laser vibrometer to measure the local strain of the friction joints. For many decades, it has been well known that nonlinear terms in dynamic equations can induce strong energy exchanges between modes (see [5], [6] and references therein). These exchanges can be also referred to modal interactions or internal resonances. When the energy of a main mode is partially transferred to its nth harmonic, it is generally denoted as a 1:n modal interaction. Modal interactions were first studied on systems with very few degrees of freedom. In [7] a 1:2 internal resonance is studied both theoretically and numerically on a two degrees of freedom system. The 1:2 modal interaction made it possible in particular to explain the undesirable roll characteristics of ships that sailors have known for centuries [8]. Many studies of modal interactions in geometrically nonlinear structures such as beams and plates can be found in [6]. Multiple mode interactions have also been studied for tuned structures such as musical instruments $([9,10,11])$. Very few studies focused on modal interactions in industrial assemblies. Recently, Noël, Renson and Kerschen $[12,13]$ highlighted modal interactions both experimentally and numerically in the vibration response of an industrial space structure where the nonlinear behavior comes from piecewise linear joints. The main contribution of the present paper is therefore to present modal interactions due to friction in an industrial assembly. These interactions that are most often unexpected in industrial studies can lead to major design issues and thus need to be studied using nonlinear dynamic methods. 
Section 2 presents the experimental results obtained exciting the "Harmony" test structure in a transversal direction. The experimental responses have been measured using both accelerometers and a 3D scanning laser vibrometer. Modal interactions are evidenced. Section 3 details the modeling methodology. Finally, section 4 presents the simulation method and compares the simulated results with the experiments.

\section{Experiments}

Experiments were performed with a shaker, accelerometers and an experimental protocol that correspond to industrial standards. In addition, a 3D scanning laser vibrometer was used to study the local slipping movement in the joints. This section presents the experimental set-up, the results obtained and focuses on modal interaction evidences.

\section{$2.1 \quad$ Experimental Set-Up}

The "Harmony" test structure is presented in detail in [1]. It is an assembly of two main parts; a central body and an external envelope the dimensions of which are presented in Table 1 . The bottom of the central body is clamped to the envelope and its top is in contact with this envelope through four blades. At high excitation level, it was demonstrated that friction appears in these contact joints [1]. In the present work, the structure is excited horizontally. The experimental set-up is presented in Fig. 1. The assembly is clamped to a moving table that is excited by an industrial shaker. The shaker is piloted in order to control the acceleration of the moving table so that the excitation level is the horizontal acceleration (along the $\mathrm{x}$ axis) of the moving table. This acceleration is measured using the accelerometer situated on the base plate (see point A10 in Fig. 1).

\begin{tabular}{llll}
\hline Part & Material & $\begin{array}{l}\text { Dimensions (mm) } \\
\text { Max radius, height }\end{array}$ & Mass (kg) \\
\hline Central Body & Stainless steel 304L & 160,300 & 11.92 \\
External envelope & Stainless steel 304L & 204, 420 & 44.88 \\
Base plate & Aluminium 2017A & 218,25 & 8.67 \\
Blade (x4) & High resistance steel & 65,118 & 0.1086 \\
& Z8-cnd17-04 & & \\
\hline
\end{tabular}

Table 1: Parts characteristics.

\subsection{Experimental results}

Firstly, swept sine experiments are realized at a low excitation level $\left(1 \mathrm{~m} \mathrm{~s}^{-2}\right)$ over a large frequency range $([5-2000] \mathrm{Hz})$. These data are used to update and validate the finite element model. The experimental modes are identified at $134.7 \mathrm{~Hz}, 309.0 \mathrm{~Hz}, 1240 \mathrm{~Hz}, 1675 \mathrm{~Hz}$ and $1826 \mathrm{~Hz}$. The experiments 


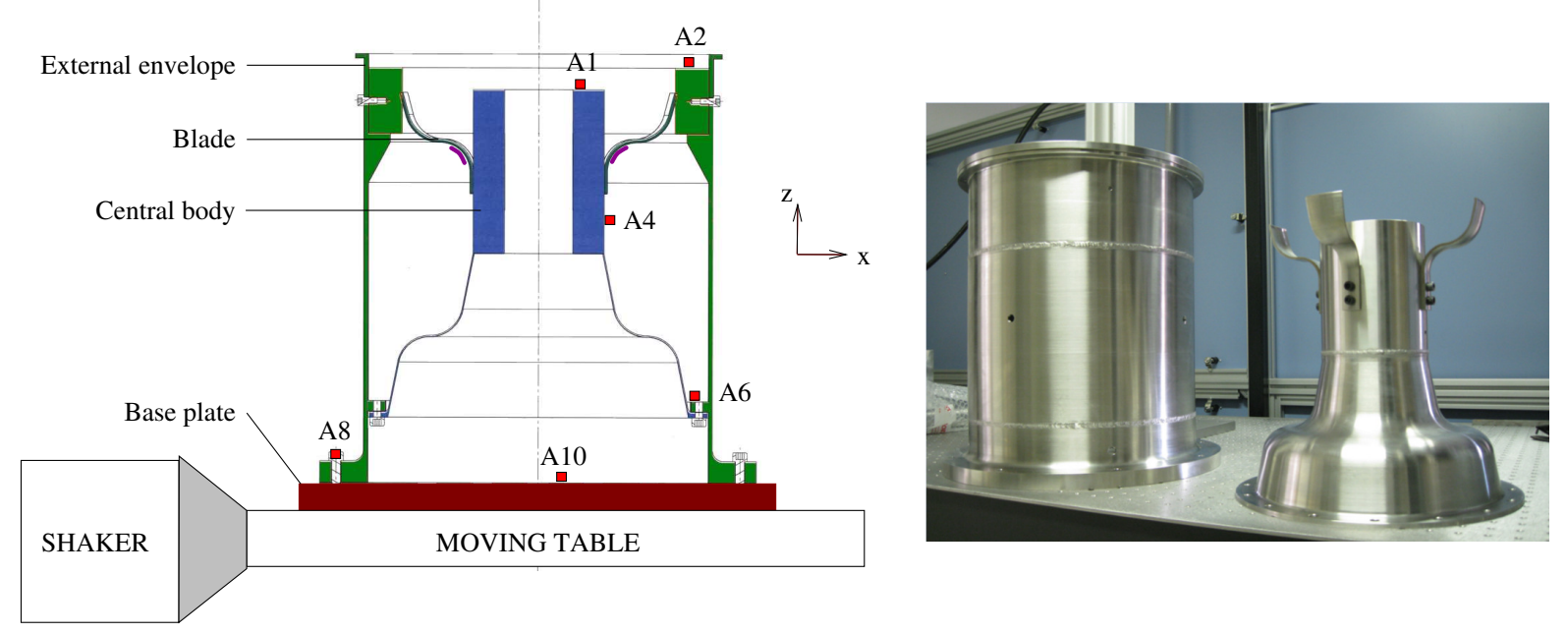

Figure 1: Experimental set-up (left) and components picture (right). Red squares represent accelerometers. Strain gages, placed under the blades, are shown in violet.

then focus on the first horizontal mode. Swept sine experiments are performed in the vicinity of this mode with increasing excitation levels. The results obtained at the top of the central body (point A1 in Fig. 1) and those obtained at the top of the external envelope (point A2 in Fig. 1) are plotted in Fig. 2. The results obtained are highly dependent on the excitation level. The resonance peak flattens and is shifted toward lower frequencies. These observations indicate increase of dissipation and a softening effect that are the main effects of friction. These effects are visible both on the central body and the envelope. Even if friction is a local phenomenon, it has a global effect on the frequency response of the structure.

\subsection{Repeatability}

For each excitation level the responses of which have been plotted in Fig. 2, the swept sine experiment was performed twice in order to appreciate the repeatability. Fig. 3 presents the results obtained in point A1 (see Fig. 1) for a $10 \mathrm{~m} \mathrm{~s}^{-2}$ excitation level. Considerable variability can be observed between the two consecutive experiments. In addition, the same experiment was performed at the end of the full experimental protocol the results of which are plotted in Fig. 2. The response obtained is compared to previous results in Fig. 3. The results appear to be different, as there appears to be a softening effect.

This poor repeatability is interpreted as both an aleatoric joint behavior during friction and as a consequence of fretting wear of the blade in the contact zone. Evidence of fretting wear was observed at the end of the experimental protocol. Fig. 4 presents two pictures of the blade condition after experiments. The black traces were created by the experiments, revealing fretting wear. For those blades the upper edge of which is collinear to the excitation direction, friction occurs on the full width 


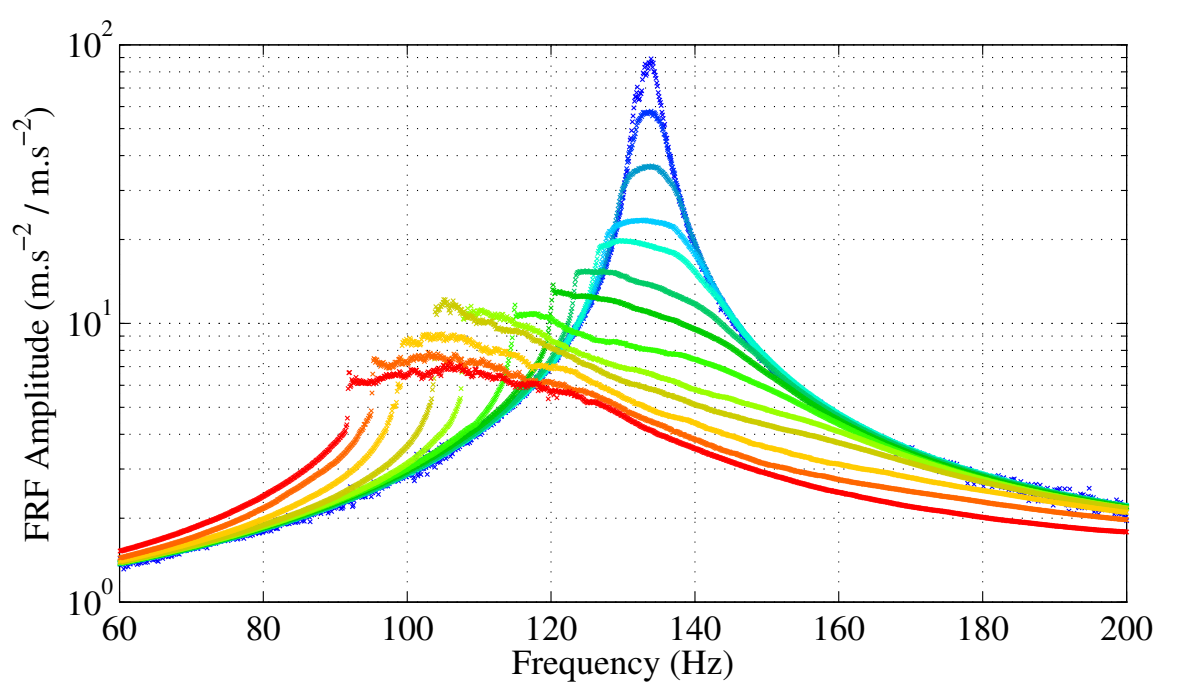

Excitation

level $\left(\mathrm{m} . \mathrm{s}^{-2}\right)$
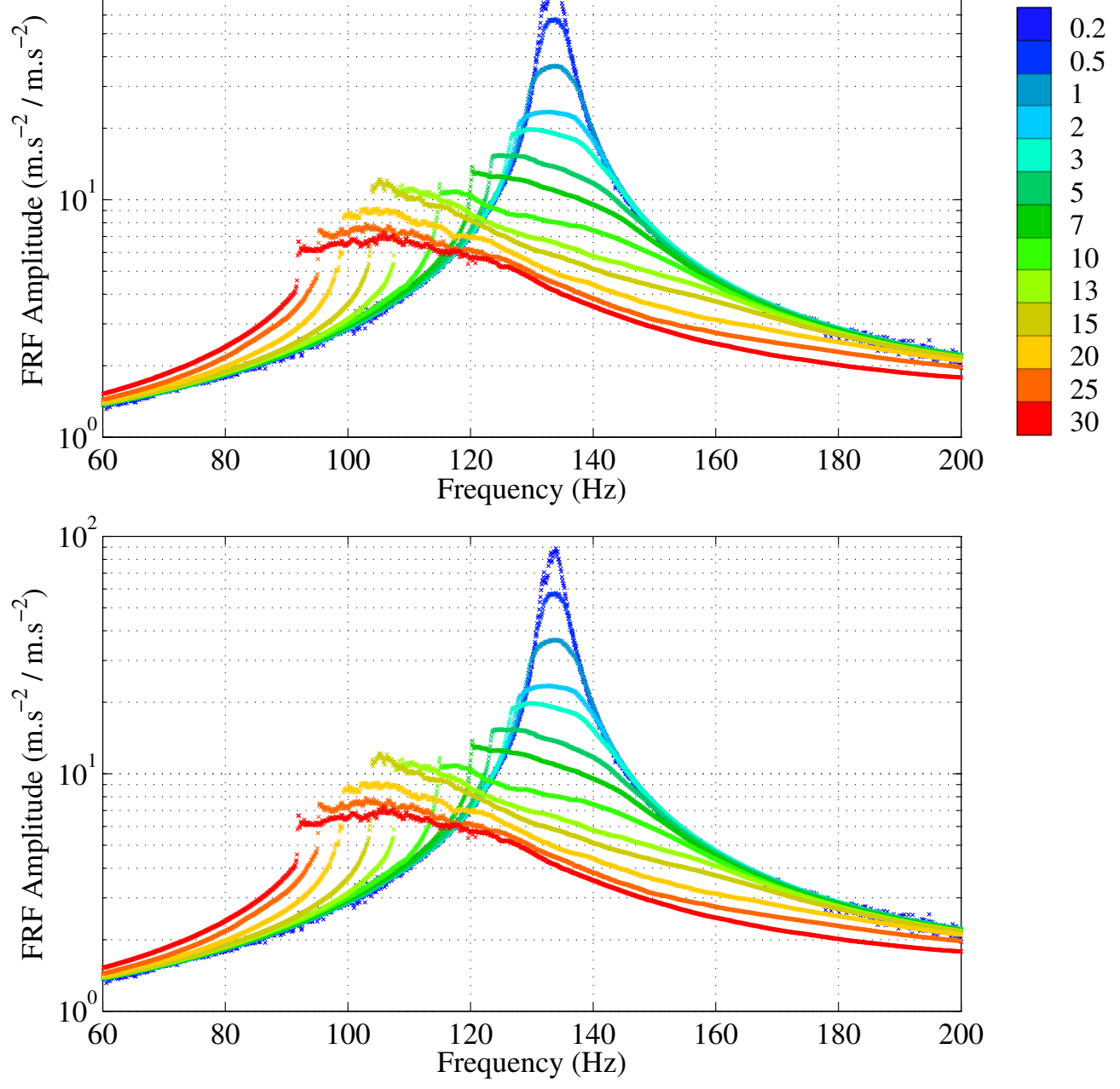

Figure 2: Experimental FRF at the top of the central body, point A1 in Fig. 1, (upper plot) and at the top edge of the external envelope, point A2 in Fig. 1, (lower plot). 


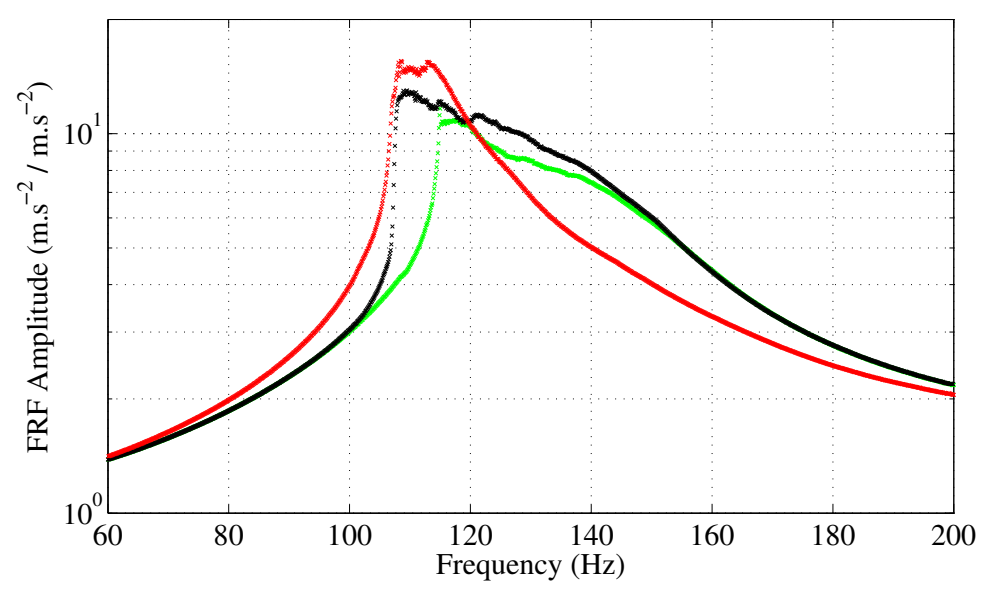

Figure 3: Repeatability. The three curves plotted are obtained with the same excitation level $\left(10 \mathrm{~m} \mathrm{~s}^{-2}\right)$. The first (green) and the second (black) correspond to consecutive measurements. The third curve (red) was obtained at the end of the full experimental protocol the results of which are plotted in Fig. 2.

of the blade (see Fig. 4, left). On the contrary, for those blades the upper edge of which is orthogonal to the excitation direction, the black traces are visible only in the vicinity of the lateral edges of the blades. This reveals a partial slipping of these blades.
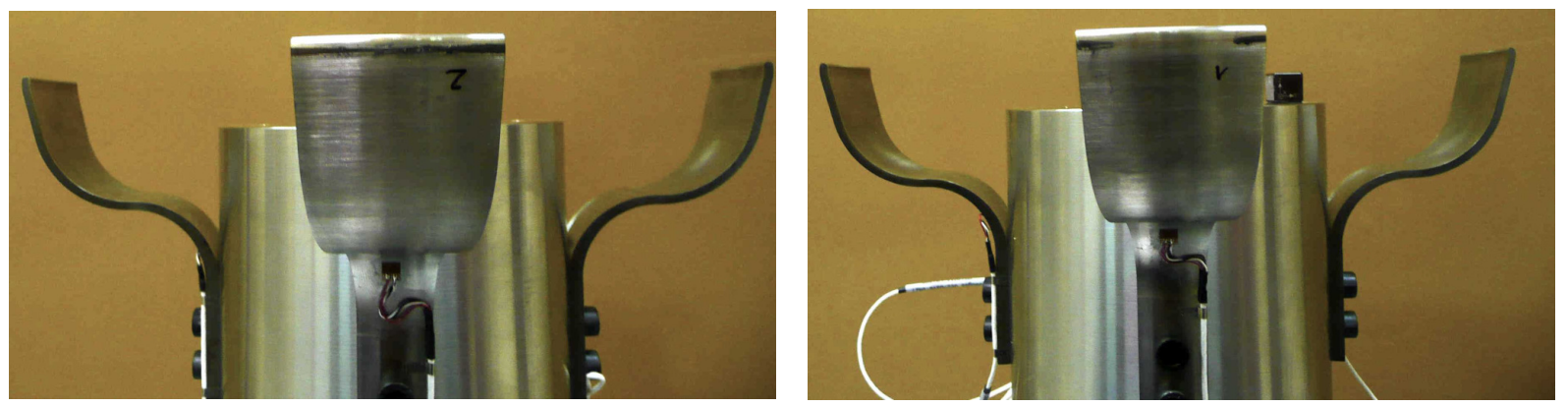

Figure 4: Pictures of the blades at the end of the experimental protocol. The picture on the left corresponds to a blade the upper edge of which is collinear to the excitation direction. The picture on the right corresponds to a blade upper edge of which is orthogonal to the excitation direction.

Abrasion of the blade can induce a softening effect in the frequency response function of the assembly. During the mounting of the assembly, the blades are compressed and this compression determines the normal contact force. When the thickness of the blade is reduced by abrasion, the normal force is also reduced. Taking a Coulomb model, the force threshold for slipping is then linearly dependent on the normal force. When the normal force is reduced, slipping is increased, and so the softening effect of friction on the frequency response function is increased. The wear of the blades due 
to experiments can thus partially explain the poor repeatability of the results.

\subsection{Modal interactions}

In these experimental results, modal interactions are observed. The main mode studied is the first transversal mode of the structure in the $\mathrm{x}$ direction (transversal with respect to the vertical symmetry axis of the test structure). When friction occurs, the energy can be transferred from this main mode to other modes of the structure. Fig. 5 presents the experimental frequency response at the top of the central body (point A1 in Fig. 1) in the transversal directions $\mathrm{x}$ and $\mathrm{y}$. As the excitation is along $\mathrm{x}$, in a linear perspective, the response along $\mathrm{y}$ should be negligible. Fig. 5 shows that in the frequency bandwidth where friction occurs $([110-150] \mathrm{Hz})$, the orthogonal response along y is considerably increased. This is interpreted as 1:1 modal interaction between the first transversal mode in the $\mathrm{x}$ direction and its twin mode in the $\mathrm{y}$ direction.

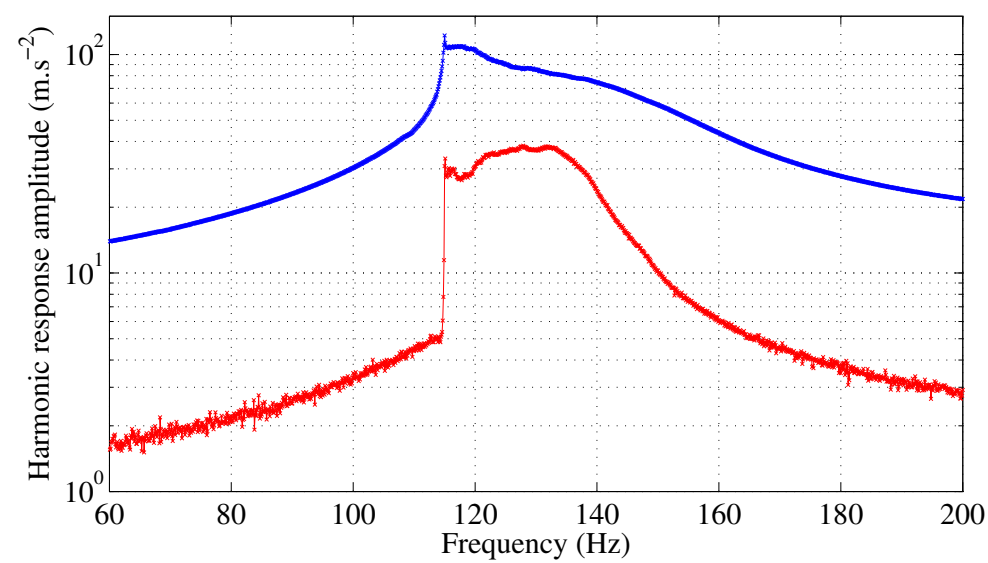

Figure 5: Experimental frequency response at the top of the central body (point A1 in Fig. 1), in the excitation direction $\mathrm{x}$ (blue curve) and in the orthogonal horizontal direction y (red curve).

To study interactions with higher order modes, a harmonic decomposition of the signal response is performed. The signal processing method used is presented in [14]. Fig. 6 presents the experimental multi-harmonic response to a $10 \mathrm{~m} \mathrm{~s}^{-2}$ excitation level. The data used for this decomposition correspond to an experiment performed after the end of the main experimental protocol. The harmonic response is that plotted in red in Fig. 3. The higher order harmonic components are at least two orders of magnitude lower than the harmonic response. In any case, in the frequency bandwidth where friction occurs $([110-150] \mathrm{Hz})$, the higher order harmonic components increase and two resonance phenomena can be highlighted : at $116 \mathrm{~Hz}$ for the 11th harmonic and at $142 \mathrm{~Hz}$ for the 9th harmonic. These two peaks both correspond to signals with a frequency of approximately $1277 \mathrm{~Hz}$ $(116 \times 11=1276 \mathrm{~Hz}$ and $142 \times 9=1278 \mathrm{~Hz})$. This frequency is close to that of the third transversal mode, measured at $1240 \mathrm{~Hz}$ before performing the experimental protocol. These two peaks can then 
be interpreted as a modal interaction between the first transversal mode and a mode located at 1277 $\mathrm{Hz}$ which may be the third transversal mode.

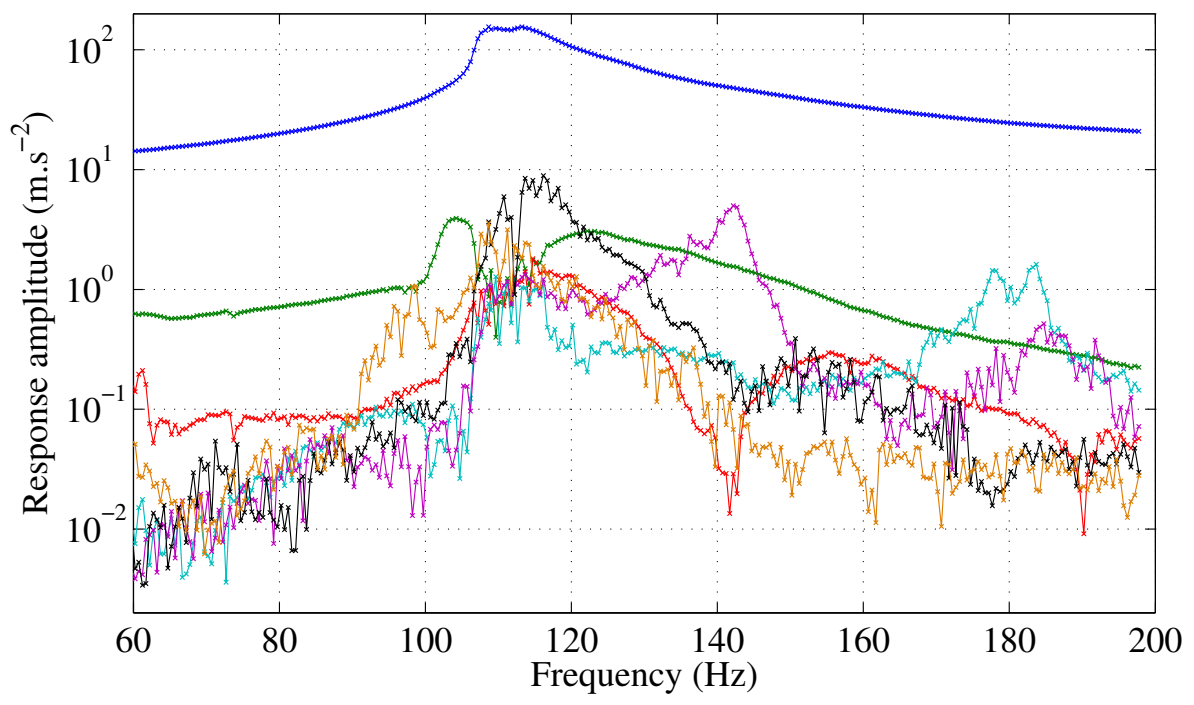

Figure 6: Multi-harmonic experimental frequency response at a $10 \mathrm{~m} \mathrm{~s}^{-2}$ excitation level. The harmonics displayed are: 1 (blue), 3 (green), 5 (red), 7 (azure), 9 (violet), 11 (black) and 13 (orange).

\subsection{Experiments with laser vibrometry}

\subsubsection{Scanning laser vibrometry}

Over and beyond the study of the assembly global response using accelerometers, the local friction movements close to the contact zone were studied. For that purpose, a 3D scanning laser vibrometer was used [15]. The experimental set-up is shown in Fig. 7. It consists of three laser measurement heads located above the assembly. One head includes a video camera which helps to position the measurement points. The location of the 163 measurement points defined for these experiments is shown in Fig. 7 (right). A $135 \mathrm{~Hz}$ fixed frequency sinusoidal excitation is applied. The stationary responses of two blades are recorded. For each measurement point, the instant velocity is measured in the three directions and recorded over a few periods (50 kHz sampling). Assuming the stationary regime, the different responses are then synchronized using the excitation signal phase. As the excitation direction is $\mathrm{x}$, the blade represented on the left in Fig. 7 is stressed in the direction of its upper edge ( $\mathrm{x}$ ) and in rotation around the $y$ axis because of the rotation of the central body. This rotation speed of the upper edge of this blade is computed from the experimental velocities measured in this area. To detail the computation of this rotation speed, the measurements points situated at the top of the blade are named according to Fig. 8. The rotation speed of the envelope and the blade are then defined by the 
following formulas:

$$
\begin{gathered}
\dot{\theta}_{y}(\text { blade })=\frac{1}{4}\left(\frac{v_{z}(A 2)-v_{z}(A 1)}{d(A 1, A 2)}+\frac{v_{z}(B 2)-v_{z}(B 1)}{d(B 1, B 2)}+\frac{v_{z}(C 2)-v_{z}(C 1)}{d(C 1, C 2)}+\frac{v_{z}(D 2)-v_{z}(D 1)}{d(D 1, D 2)}\right) \\
\dot{\theta}_{y}(\text { envelope })=\frac{v_{z}(E 2)-v_{z}(E 1)}{d(E 1, E 2)}
\end{gathered}
$$

where $v_{z}$ is the velocity measured in the $\mathrm{z}$ direction and $d$ is the distance between two points.
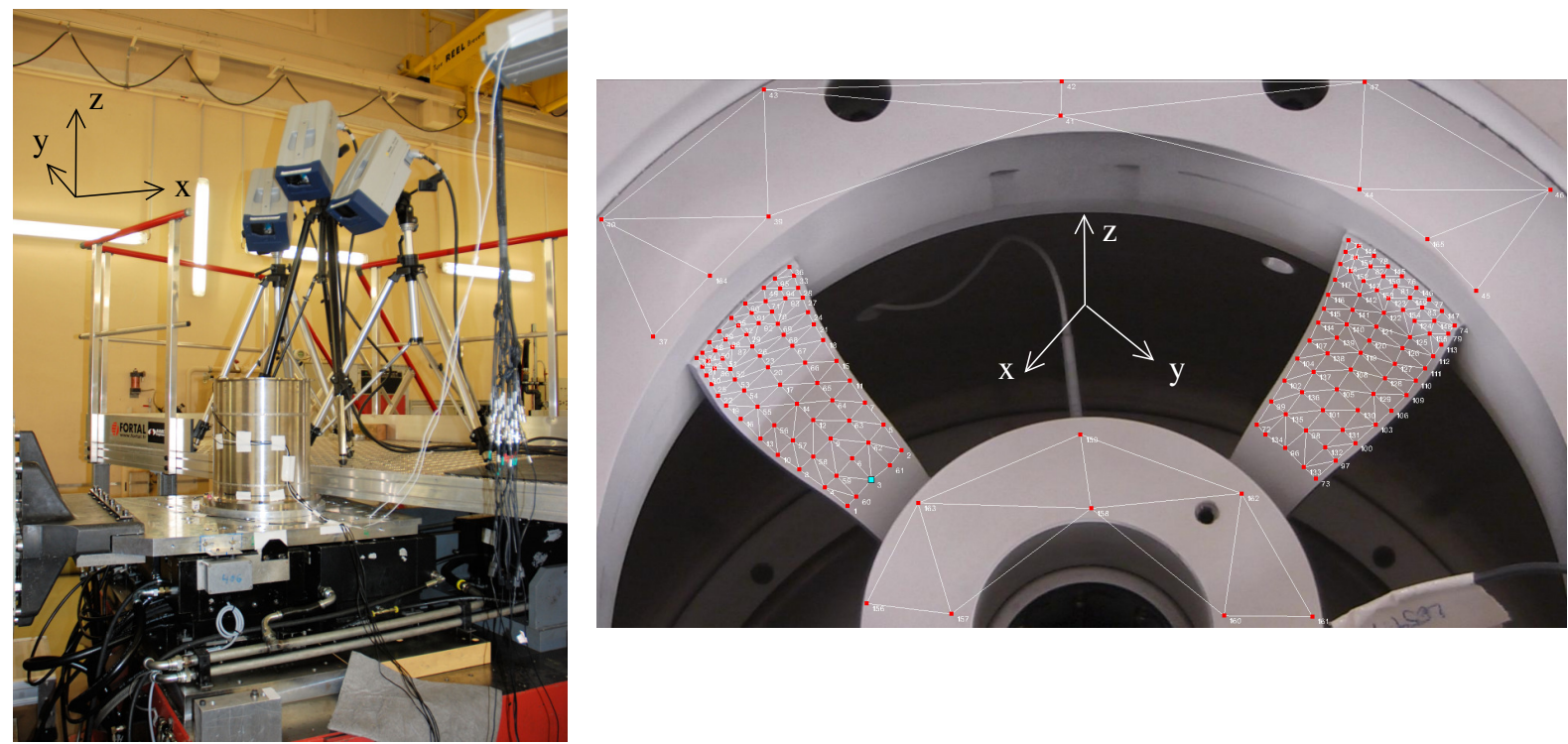

Figure 7: 3D scanning laser vibrometry set-up (left) and location of the measurement points (right).

The experimental results thus computed are plotted in Fig. 9. They correspond to a very low excitation: level of $0.5 \mathrm{~m} \mathrm{~s}^{-2}$. As with the vibrometry measurements presented in [1], Fig. 9 shows an alternation of stick and slip periods which evidence friction. When the two curves overlap, the blade and the envelope have the same movement. The blade remains stuck to the envelope. The separation of the two curves reveals slipping. Even at such a low excitation level, the blade undergoes rotational friction. Similar results show that at a higher excitation level this blade also undergoes translation friction in the $\mathrm{x}$ direction. Then again at higher excitation level, the other blade studied (oriented in an orthogonal direction) undergoes vertical friction.

\subsubsection{Modal interaction with a blade mode}

Focusing on rotational friction, at a higher excitation level, the results observed are very different from those presented in Fig. 9. Fig. 10 shows the time recording of the rotational speed responses of the envelope and the blade to a $10 \mathrm{~m} \mathrm{~s}^{-2}$ excitation level. Slip and stick periods can still be distinguished but considerable oscillations of the rotational speed of the blade are observed during the slip periods. For a better understanding of this phenomenon, the deformed shape measured by the vibrometer is 


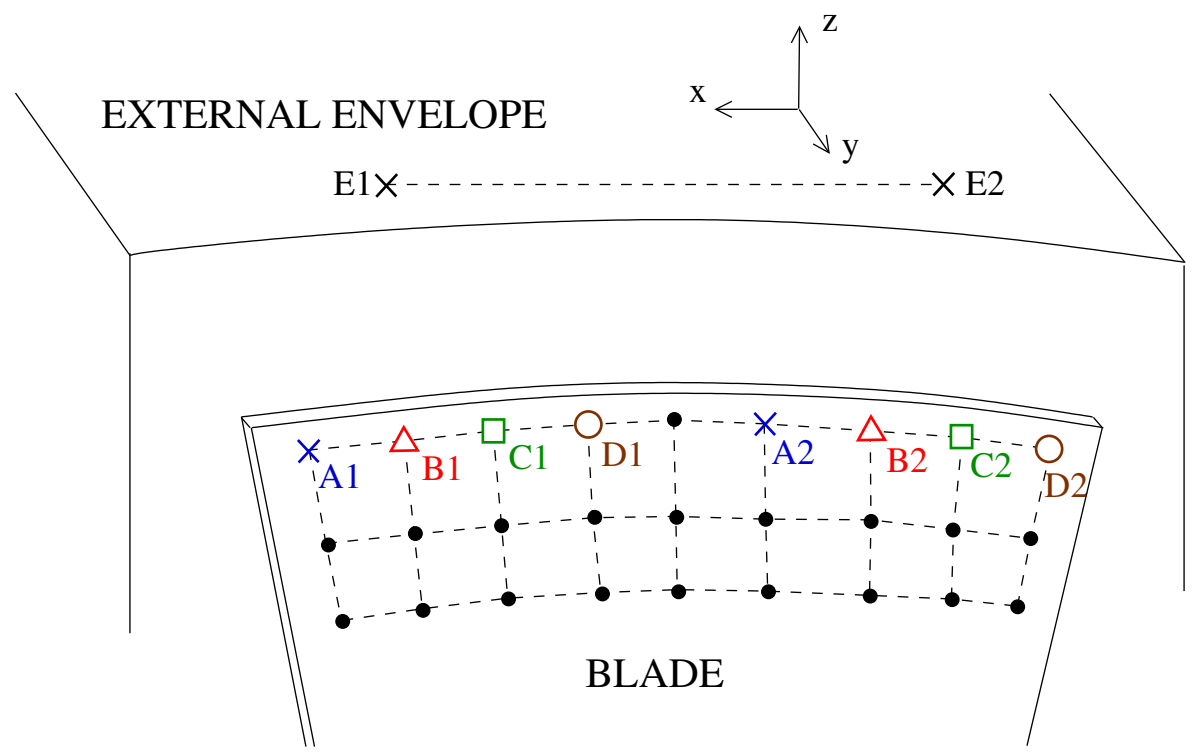

Figure 8: Notation scheme for the computation of the blade rotational speed.

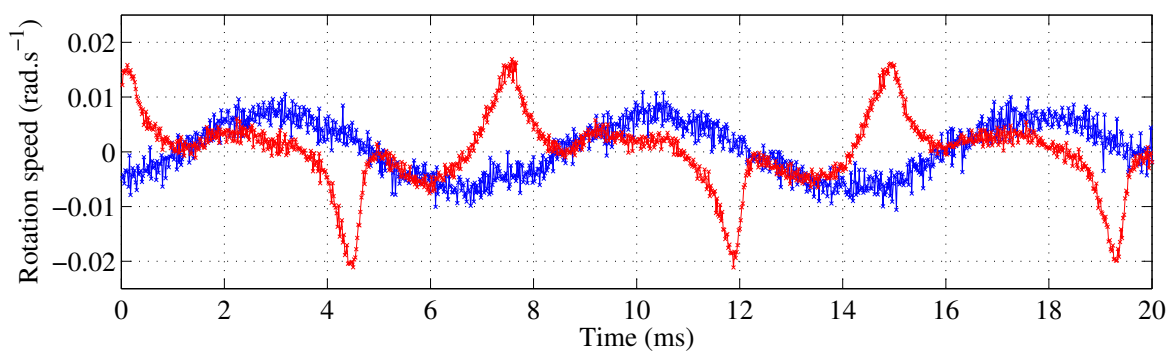

Figure 9: Experimental rotational velocity around the y axis of the envelope (blue) and the top of the blade (red). 


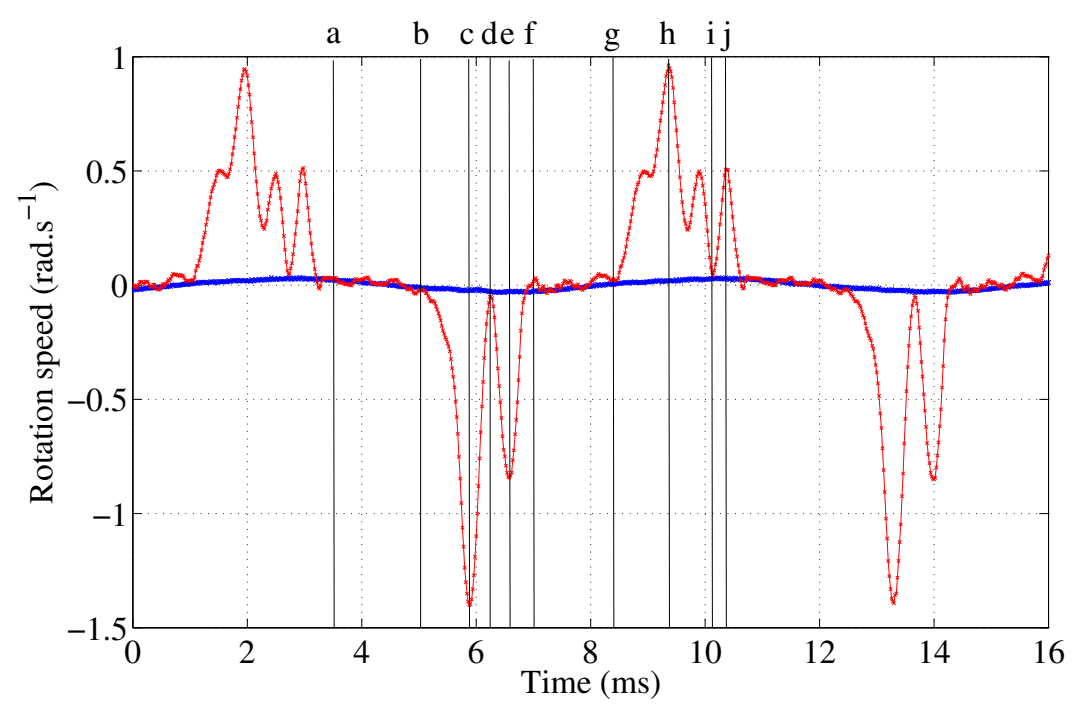

Figure 10: Experimental rotation velocities around the y axis of the envelope (blue) and the upper edge of the blade (red). The excitation level is $10 \mathrm{~m} \mathrm{~s}^{-2}$.

presented in Fig. 11. Each picture corresponds to an instant that is defined in Fig. 10 (see markers $\mathrm{a}, \mathrm{b}, \mathrm{c}, \ldots)$. The experimental mesh is colored and deformed according to the velocity measurements. The color represents the norm of the measured speed vector, from blue (zero speed) to red (maximal speed). The experimental points are displaced according to the velocity vector measured.

The pictures (b, c, d, e and f) indicate a large rotational oscillation of the blade. The frequency of this oscillation is around $1750 \mathrm{~Hz}$, which is close to the frequency of the 13th harmonic of the response signals. The signal processing software used to display the vibrometry measurements (Polytec ScanViewer) can display individually each harmonic component of the measured signal. The deformed shape corresponding to the 13 th harmonic (at $135 \times 13=1755 \mathrm{~Hz}$ though) is shown in Fig. 12. This deformed shape corresponds to a blade pumping mode. This indicates that the large oscillations observed in Fig. 10 must be due to a modal interaction between the main transversal mode and a blade pumping mode the resonance frequency of which must be approximately $1755 \mathrm{~Hz}$.

This hypothesis is confirmed by the study of the mean displacement of the blade in the y direction. Fig. 13 presents the time record of the mean velocity of the blade in the y direction (i.e. the mean value of the instant velocity in the y direction of all the measurement points located on the blade). Two results are presented. They correspond to the same excitation level but with two different excitation frequencies: $130 \mathrm{~Hz}$ and $135 \mathrm{~Hz}$. The main component of the measured signal is the first harmonic but at $135 \mathrm{~Hz}$, the signal is considerably perturbed by a 13th harmonic component. This modal interaction which is obvious at $135 \mathrm{~Hz}$, vanishes at $130 \mathrm{~Hz}$ where the 13 th harmonic frequency is 1690 Hz. This confirms the hypothesis of a modal interaction that can only occur when there is an integer ratio between the excitation frequency and the resonance frequency of the mode involved. 

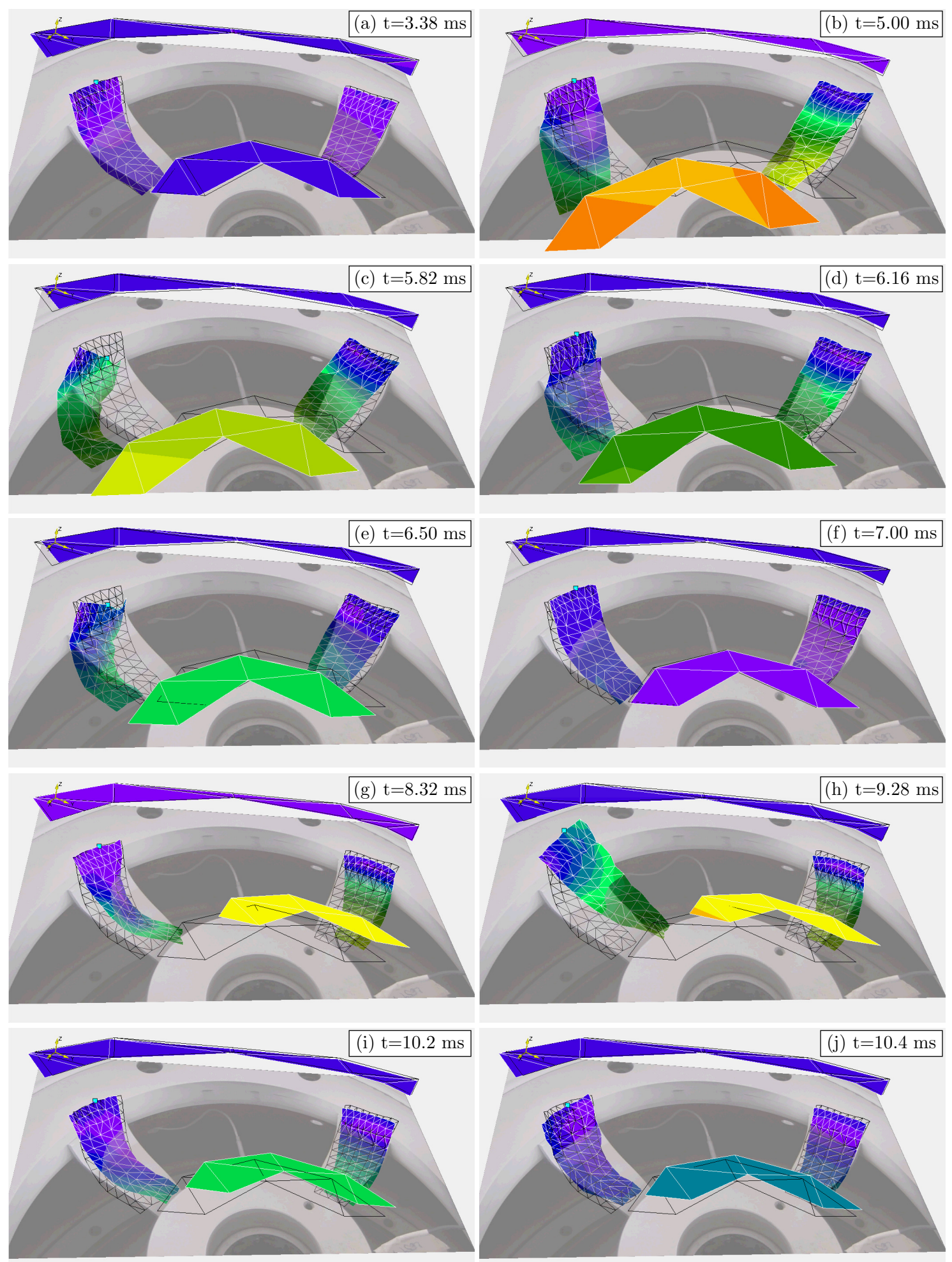

Figure 11: Images corresponding to the velocities measured in the contact zone by the vibrometer at different instants in an excitation cycle. The instants are named in Fig. 10. 


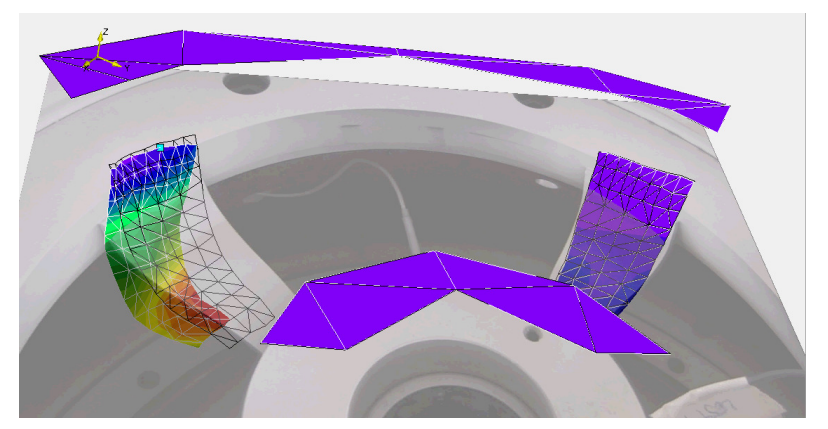

Figure 12: 13th harmonic component of the velocity signals measured by the 3D scanning vibrometer.
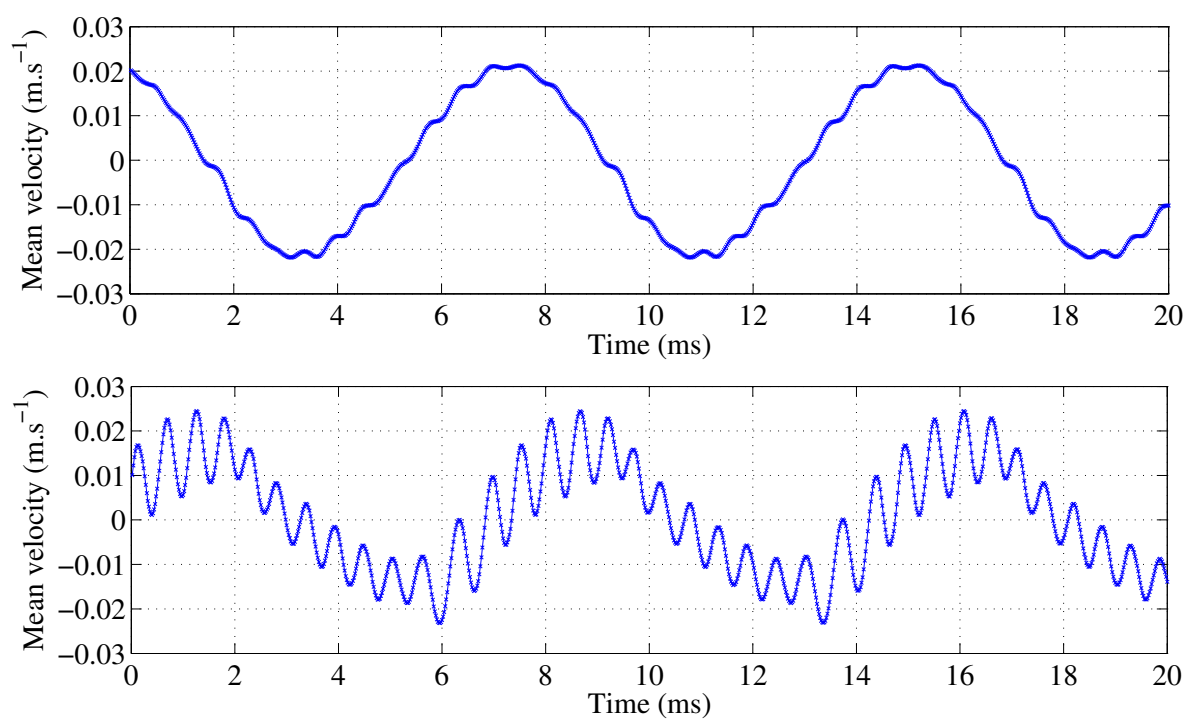

Figure 13: Mean velocity of the blade in the y direction. The excitation frequencies are $130 \mathrm{~Hz}$ (upper plot) and $135 \mathrm{~Hz}$ (lower plot). The excitation amplitude is $10 \mathrm{~m} \mathrm{~s}^{-2}$ in both cases. 


\begin{tabular}{lll}
\hline $\begin{array}{l}\text { Numerical resonance } \\
\text { frequency }\end{array}$ & $\begin{array}{l}\text { Frequency error } \\
\text { with respect to } \\
\text { experiments }\end{array}$ & $\begin{array}{l}\text { Spatial } \\
\text { correlation } \\
\text { (MAC) }\end{array}$ \\
\hline $133.4 \mathrm{~Hz}$ & $-1.0 \%$ & 94.4 \\
$308.5 \mathrm{~Hz}$ & $-0.2 \%$ & 98.4 \\
$1212 \mathrm{~Hz}$ & $-2.3 \%$ & 87.1 \\
\hline
\end{tabular}

Table 2: Comparison between numerical and experimental eigenmodes.

\section{Modeling}

Considering these experimental results, nonlinear modeling of the structure appears to be necessary to be able to simulate the dissipation, softening and modal interaction effects. The modeling developed is very similar to that developed in [1]. The assembly considered in its experimental set-up is modeled with finite elements. This model is reduced using a substructuring algorithm. Finally, the linear joint models are replaced by nonlinear Coulomb models.

\subsection{Finite Element Model}

The finite element model of the assembly has been previously developed in [1]. In this previous work, the model was validated component by component using dedicated experiments. Here, this model has only been adapted to the experimental set-up. The finite element model is presented in Fig. 14. The shaker and the moving table are not modeled. The assembly is considered in the moving plate reference frame. The excitation is applied as a gravity force resulting from the absolute acceleration of the frame. A reference point "Ref_base", fixed in this frame is created. The degrees of freedom (DOF) corresponding to the lower face of the base plate are all driven by the reference point "Ref_base" through a kinematic coupling. As this reference point is fixed in the moving table reference frame, its DOFs in the directions $\mathrm{x}, \mathrm{y}, \mathrm{z}$ and in rotation around the $\mathrm{z}$ axis are blocked. Its rotational DOFs around $\mathrm{x}$ and $\mathrm{y}$ axis are not blocked. They are connected to the ground through two rotational springs the stiffness values of which are updated: $k_{\text {"ref base" } x}=k_{\text {"ref base" } y}=3 \mathrm{e} 7 \mathrm{~N} \mathrm{rad}^{-1}$. These non-ideal boundary conditions represent the rotational stiffness of the moving plate that is simply supported on an oil bath. They considerably improve the agreement between numerical and experimental eigenfrequencies.

Experimental and numerical modal analyzes were performed. The comparison between the numerical and experimental modes is presented in Table 2. The linear vibration response of the finite element model is compared to the experimental results at a low excitation level in Fig. 15. For this computation, a set of modal damping coefficients identified from experiments was introduced in the numerical model.

There is a very good agreement between the numerical and the experimental frequency response 

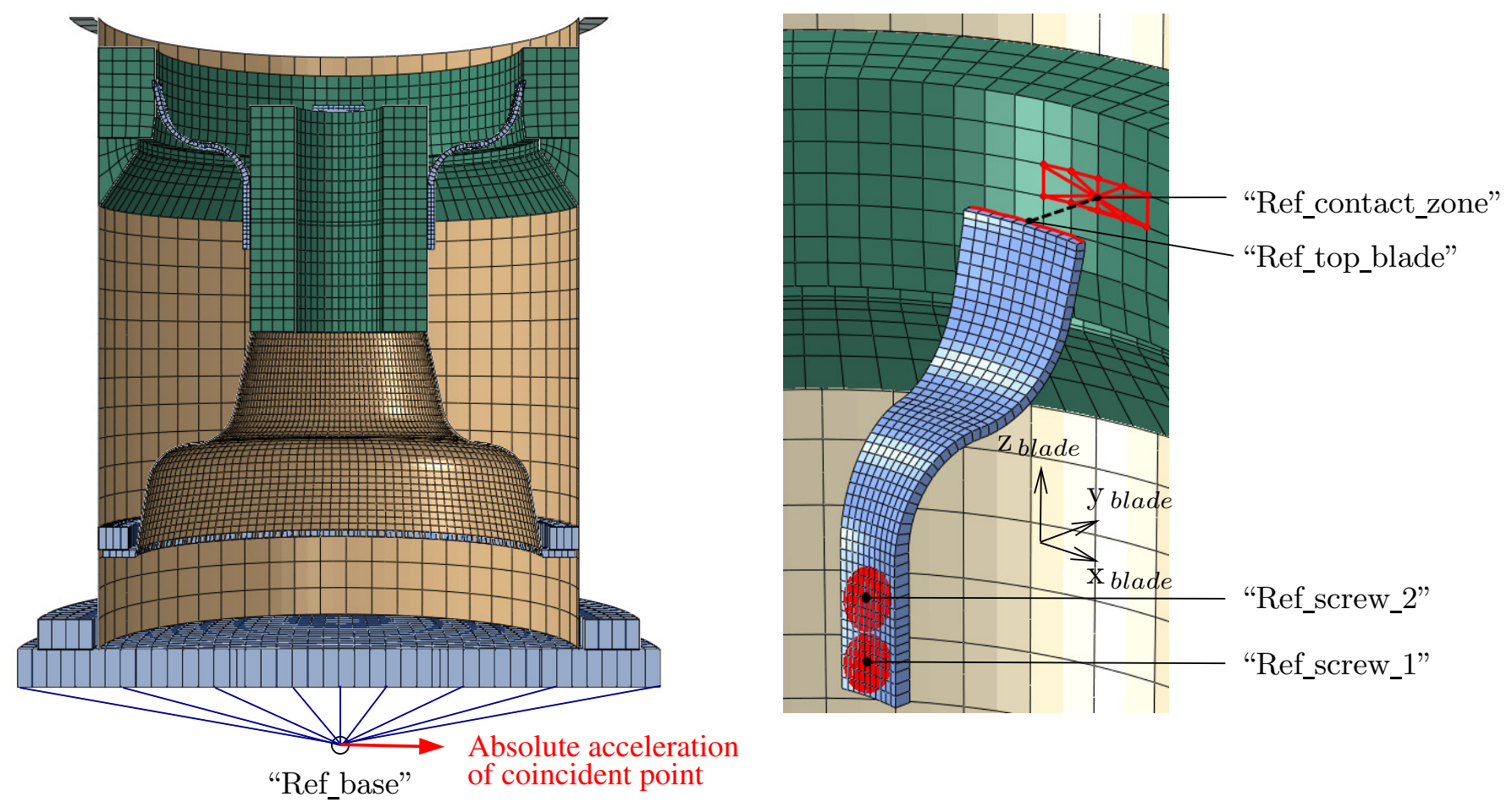

Figure 14: Finite Element model of the assembly (left) and focus on the modeling of the blades (right). 

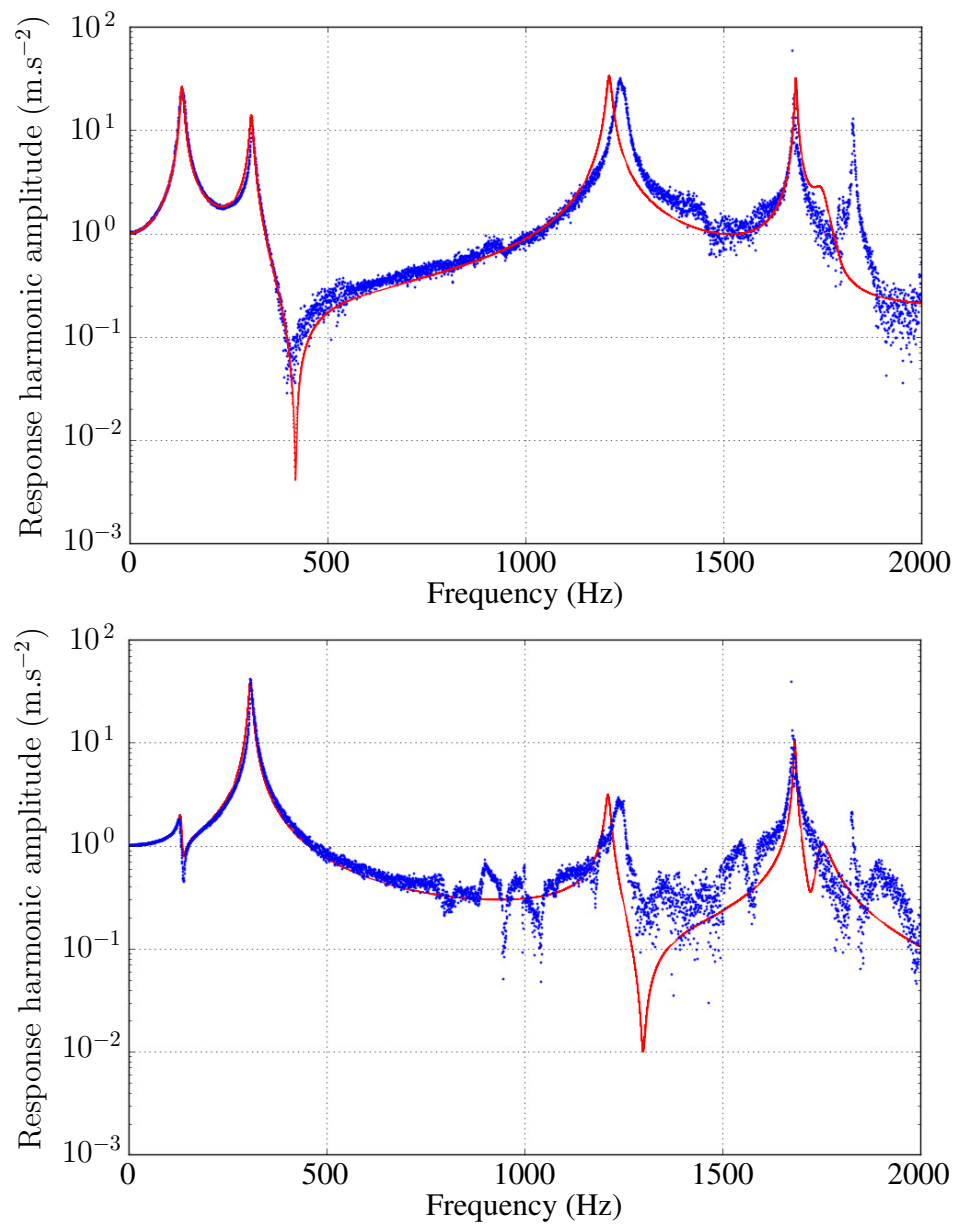

Figure 15: Comparison between the experimental (blue dots) and numerical (red curve) frequency response to a $1 \mathrm{~m} \mathrm{~s}^{-2}$ excitation level. The upper plot presents the results obtained at the top of the central body (point A1 in Fig. 1) in and the lower plot those obtained at the top of the external envelope (point A2 in Fig. 1) 
over a large frequency band. The structure is then only excited in the vicinity of the first transversal mode but Section 2 showed that modal interactions can nevertheless involve modes situated at a much higher frequency. A finite element model updated for a very large frequency range is then necessary to make nonlinear simulation capable of predicting modal interactions.

\subsection{Model reduction}

The finite element model introduced in Section 3.1 includes 174054 DOFs. In order to lighten the computational burden associated with nonlinear simulations, the model is reduced using a substructuring algorithm. The method used has already been presented in a previous work [1]. It uses a substructuring algorithm implemented in Abaqus [2] that is very similar to the Craig and Bampton method [16] except that it ensures that the eigenmodes of the reduced model are exactly those retained from the full model. The reduction basis is divided into static vectors $\boldsymbol{\Psi}_{\mathbf{r}}$ and dynamic vectors $\boldsymbol{\Phi}_{\alpha}$. The reduction basis is then $\left[\mathbf{\Psi}_{\mathbf{r}}, \mathbf{\Phi}_{\alpha}\right]$. The static vectors are introduced to retain physical DOFs from the full finite element model. The DOFs of the nodes corresponding to the location of the accelerometers are retained to be able to perform direct comparisons with experiments. For each blade, three nodes are retained to be able to introduce a nonlinear joint model between them. The nodes retained are "Ref_screw_1", "Ref_screw_2" and "Ref_contact_zone" according to Fig. 14 notations. "Ref_top_blade" is deliberately not retained because previous works have shown that the movement of this node is strongly non-harmonic and retaining it induces major convergence issues in the nonlinear simulation process. Consequently, the workaround logic introduced in [1] is kept for this study.

In addition, the 30 first eigenmodes of the full finite element model are retained through dynamic vectors. By so doing, the linear dynamic behavior of the full and reduced model, measured in the retained DOFs, is exactly the same over the entire [0-2000] Hz frequency range. The dynamic response of the reduced model is thus in very good agreement with the experiment at a low excitation level in this frequency range.

The linear dynamic equation is written in this reduced basis:

$$
\mathbf{M} \ddot{\mathbf{U}}+\mathbf{D} \dot{\mathbf{U}}+\mathbf{K U}=\mathbf{F}_{\text {excit }} \cos (\Omega t)
$$

$\mathbf{M}$ and $\mathbf{K}$ are the mass and stiffness reduced matrices. $\mathbf{U}$ and $\mathbf{F}_{\text {excit }}$ are respectively the displacement and excitation force vectors in the reduced basis. The damping matrix $\mathbf{D}$ is defined as proportional to the mass matrix: $\mathbf{D}=\alpha \mathbf{M}$ where alpha is updated in order to fit the experimental modal damping for the first transversal resonance. This damping model has been chosen for its ease of implementation. Several experimental modal damping coefficients may be taken into account by using Caughey series.

\section{$3.3 \quad$ Joint modeling}

Once the reduced model has been defined, the nonlinear joint equations can be introduced. The first step of this introduction is the definition of the application vectors. In the full finite element 
model, the joints are modeled using strong stiffness connectors between nodes "Ref_top_blade" and "Ref_Contact_Zone" (see Fig. 14). The components of these connectors are the linear joints that are replaced by friction models. Each component of a connector has a stiffness $k_{\text {joint full model }}$ and defines an application vector $\mathbf{V}_{\text {joint full model }}$. In order to avoid definition mistakes, the following verification can be performed computing the difference between the stiffness matrix of the full model and the stiffness matrix of the same model assembled without the joint:

$$
\mathbf{K}_{\text {full model }}-\mathbf{K}_{\text {full model without joint }}=k_{\text {joint full model }} \mathbf{V}_{\text {joint full model }}{ }^{T} \mathbf{V}_{\text {joint full model }}
$$

where ${ }^{T}$ denotes the transposed vector. Once the joint stiffness and the application vectors have been defined in the full model, they are projected on the reduced model:

$$
\begin{gathered}
\mathbf{V}_{\text {joint }}={ }^{T}\left[\boldsymbol{\Psi}_{\mathbf{r}}, \boldsymbol{\Phi}_{\alpha}\right] \mathbf{V}_{\text {joint full model }} \\
k_{\text {joint }}=k_{\text {joint full model }} \frac{\left\|\mathbf{V}_{\text {joint full model }}\right\|^{2}}{\left\|\mathbf{V}_{\text {joint }}\right\|^{2}}
\end{gathered}
$$

The projection of the displacement on the application vector is denoted $u_{\text {joint }}$ :

$$
u_{\text {joint }}={ }^{T} \mathbf{V}_{\text {joint }} \mathbf{U}
$$

This linear model of the joint is replaced by an elastic Coulomb model which consists of a Coulomb slider and a spring in series. This model that may equally be called the 1-dimensionnal Masing model, Jenkins element or Prandtl model, is presented in Fig. 16a. The only parameter of the model is the Coulomb threshold $F_{\text {Coulomb joint }}$. The friction force is computed using the iterative formulation introduced by Guillen and Pierre [17] and already chosen in [1]. For each time iteration i, in a first step, a trial force is estimated assuming that the friction slider remains in the stuck position between instants $t_{i-1}$ and $t_{i}$ :

$$
{ }^{t r} f=f_{\text {joint }}\left(t_{i-1}\right)+k_{\text {joint }}\left(u_{\text {joint }}\left(t_{i}\right)-u_{\text {joint }}\left(t_{i-1}\right)\right)
$$

Then if the trial force remains below the Coulomb threshold $F_{\text {Coulomb joint }}$, the stuck assumption is validated; the contact behavior is purely elastic. Otherwise, a classic friction behavior is assumed:

$$
f_{i}=\left\{\begin{array}{lll}
{ }^{t r} f & \text { if }\left|{ }^{t r} f\right|<F_{\text {Coulomb joint }} & \text { stick } \\
\operatorname{sgn}\left({ }^{t r} f\right) F_{\text {Coulomb joint }} & \text { if }\left|{ }^{t r} f\right| \geq F_{\text {Coulomb joint }} & \text { slip }
\end{array}\right.
$$

Once applied to a periodic motion, the force-displacement diagram depicted in Fig. 16b is obtained.

In the presented model there are 4 contact zones and each has 6 degrees of freedom. There could be up to 24 friction joints introduced. The number of nonlinear joint models has been reduced to the number of nonlinear degrees of freedom actually subjected to friction. The vibrometry measurements presented in Section 2.5.1 have shown that slipping occurs in only 6 degrees of freedom. For the two blades upper edge of which is collinear to the excitation direction, slipping occurs in rotation around the $\mathrm{y}$ axis and in the $\mathrm{x}$ direction. For other two blades, slipping occurs only in the vertical z direction. 


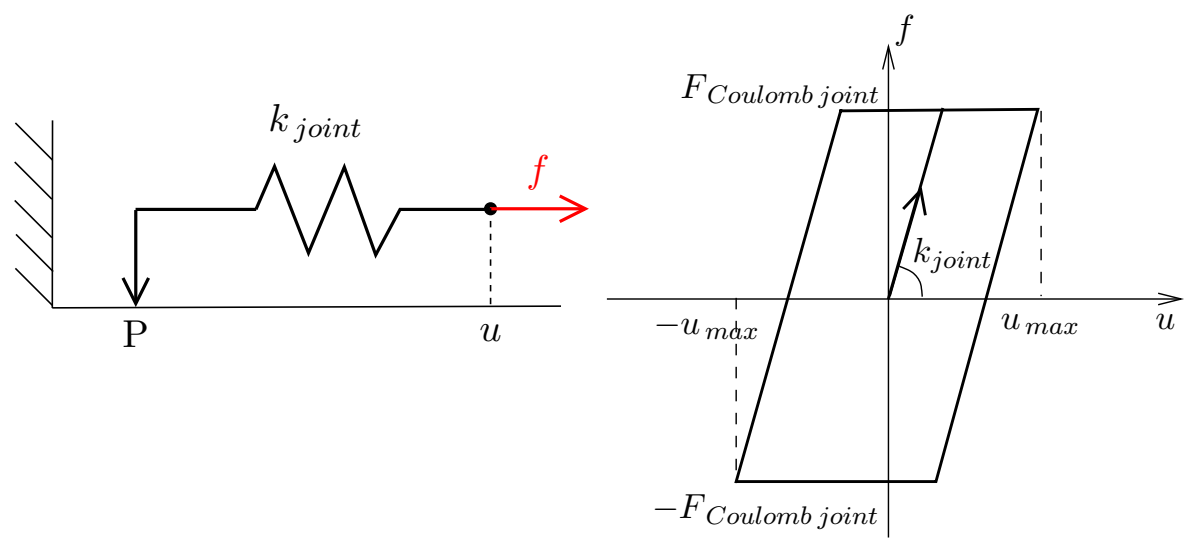

(a)

(b)

Figure 16: Scheme (a) and force displacement diagram (b) of the elastic Coulomb model

These are the 6 nonlinear joints introduced in the numerical model. Due to the symmetry, they involve 3 different parameters: $F_{\text {Coulomb } \theta y}, F_{\text {Coulombx }}$ and $F_{\text {Coulombz }}$.

At time $t_{i}$, the nonlinear force associated with each joint is computed and the total nonlinear force is obtained by summing all the contributions. As the elastic Coulomb model replaces the linear model, the nonlinear force for each joint is the difference between the linear and the nonlinear model:

$$
\mathbf{F}_{\mathbf{n l}}\left(t_{i}\right)=\sum_{\text {joint }}\left(k_{\text {joint }} u_{\text {joint }}-f_{\text {joint }}\left(t_{i}\right)\right) \mathbf{V}_{\text {joint }}
$$

The nonlinear dynamic equation is then:

$$
\mathbf{M} \ddot{\mathbf{U}}+\mathbf{D} \dot{\mathbf{U}}+\mathbf{K U}=\mathbf{F}_{\text {excit }} \cos (\Omega t)+\mathbf{F}_{\mathbf{n l}}(\mathbf{U})
$$

The modeling method implemented associates uncoupled friction models to some application vectors. These application vectors correspond to selected deformed shapes of each blade. The modeling method is thus similar to that proposed by Festjens [18]. Festjens propose to define a set of deformed shapes for each joint called the "Principal Joint Strains Basis" and then to associate to each vector a nonlinear model. In the model presented above, only the deformed shapes where the upper edge of the blade remains rigid are retained. In any case, vibrometry measurements have shown that the upper edge of the blade can be deformed. One way to improve the modeling could be to associate new nonlinear models to deformed shapes of the blade where its upper edge is deformed. Another way to improve the model could be to replace the elastic Coulomb models chosen by friction models which include partial slip or micro slip. Among these models there are for example the Dahl model [19], the Iwan model [20], the Valanis model [21] and the LuGre friction model [22]. 


\section{Nonlinear simulations}

Once the nonlinear dynamic equation has been established, the Harmonic Balance Method with a condensation process and a continuation method is used. This simulation process is briefly presented in Section 4.1. The simulated results can then be compared with experimental results (Section 4.2). Finally, Section 4.3 focuses on the evidence of modal interaction in the numerical results.

\subsection{Simulation method}

The Harmonic Balance Method [3] is implemented. As the excitation term is periodic, it is assumed that the nonlinear dynamic response and the force vector may be approximated by finite Fourier series with $\Omega$ as the fundamental frequency.

$$
\begin{gathered}
\mathbf{U}(t)=\mathbf{B}_{0}+\sum_{k=1}^{N}\left(\mathbf{B}_{k} \cos (k \Omega t)+\mathbf{A}_{k} \sin (k \Omega t)\right) \\
\mathbf{F}_{\mathbf{n l}}(t)=\mathbf{C}_{0}+\sum_{k=1}^{N}\left(\mathbf{C}_{k} \cos (k \Omega t)+\mathbf{S}_{k} \sin (k \Omega t)\right)
\end{gathered}
$$

$\mathrm{N}$, the order of the Fourier series is selected on the basis of the number of significant harmonics expected in the nonlinear dynamic response. For our application, $\mathrm{N}=15$ has been chosen. Then, considering the fact that $\mathbf{F}_{\text {excit }}(t)=\mathbf{C}_{1 \text {, excit }} \cos (\Omega t)$, the equation (11) is rewritten in the Fourier basis:

$$
\begin{aligned}
& \mathbf{K B}_{0}=\mathbf{C}_{0} \\
& {\left[\begin{array}{cc}
\mathbf{K}-\Omega^{2} \mathbf{I}_{d} & -\Omega \mathbf{D} \\
\Omega \mathbf{D} & \mathbf{K}-\Omega^{2} \mathbf{I}_{d}
\end{array}\right]\left[\begin{array}{l}
\mathbf{A}_{1} \\
\mathbf{B}_{1}
\end{array}\right]=\left[\begin{array}{l}
\mathbf{S}_{1} \\
\mathbf{C}_{1}
\end{array}\right]+\left[\begin{array}{c}
0 \\
\mathbf{C}_{1, \text { excit }}
\end{array}\right]} \\
& {\left[\begin{array}{cc}
\mathbf{K}-(k \Omega)^{2} \mathbf{I}_{d} & -k \Omega \mathbf{D} \\
k \Omega \mathbf{D} & \mathbf{K}-(k \Omega)^{2} \mathbf{I}_{d}
\end{array}\right]\left[\begin{array}{l}
\mathbf{A}_{k} \\
\mathbf{B}_{k}
\end{array}\right]=\left[\begin{array}{c}
\mathbf{S}_{k} \\
\mathbf{C}_{k}
\end{array}\right] \quad \forall k \in[2, N]}
\end{aligned}
$$

where $\mathbf{I}_{d}$ is the identity matrix.

The coefficients $\mathbf{C}_{0}, \mathbf{S}_{k}$ and $\mathbf{C}_{k}$ are calculated by applying the Alternating Frequency Time domain method (AFT-method) that has been presented by Cameron and Griffin [23]. This method is summed up by the following scheme, where DFT means Discrete Fourier Transform:

$$
\mathbf{X}=\left[\mathbf{B}_{0} \mathbf{A}_{1} \mathbf{B}_{1} \ldots \mathbf{A}_{N} \mathbf{B}_{N}\right] \stackrel{D F T^{-1}}{\rightarrow} \mathbf{U}(t) \rightarrow \mathbf{F}(\mathbf{U}, t) \stackrel{D F T}{\rightarrow}\left[\mathbf{C}_{0} \mathbf{S}_{1} \mathbf{C}_{1} \ldots \mathbf{S}_{N} \mathbf{C}_{N}\right]
$$

The Discrete Fourier Transform has been performed using 300 time steps over an excitation period. Then, the dynamic equation in the Fourier basis can be rewritten:

$$
\mathbf{A} \mathbf{X}=\mathbf{W}_{\text {excit }}+\mathbf{W}_{\mathbf{n l}}(\mathbf{X})
$$

with 


$$
\begin{aligned}
& \mathbf{A}=\operatorname{Diag}\left(\mathbf{K},\left\{\left[\begin{array}{cc}
\mathbf{K}-(k \Omega)^{2} \mathbf{I}_{d} & -k \Omega \mathbf{D} \\
k \Omega \mathbf{D} & \mathbf{K}-(k \Omega)^{2} \mathbf{I}_{d}
\end{array}\right]\right\}_{k=1, N}\right) \\
& \mathbf{W}_{\text {excit }}=\left[\begin{array}{lllll}
\mathbf{0} & \mathbf{0} & \mathbf{C}_{1, \text { excit }} & \mathbf{0} & \ldots
\end{array}\right]_{2 N+1} \\
& \mathbf{W}_{\mathbf{n l}}(\mathbf{X})=\left[\mathbf{C}_{0} \mathbf{S}_{1} \mathbf{C}_{1} \ldots \mathbf{S}_{N} \mathbf{C}_{N}\right]_{2 N+1}
\end{aligned}
$$

A condensation process is introduced in order to separate nonlinear DOFs from linear ones. Such a condensation was originally proposed by Hahn and Chen [24] and has been used in an industrial context by Sinou [4]. The formulation used in this work is that detailed in [1]. The number of nonlinear joints is denoted $q(q=6)$. These $q$ application vectors $\mathbf{V}_{\mathbf{q}}=\left\{\mathbf{V}_{i}\right\}_{i=1 . . q}$ are selected to be the first vectors of a new basis. The basis is obtained using a Gram-Schmidt orthonormalisation algorithm. In this new basis $\left[\tilde{\mathbf{V}}_{\mathbf{q}}, \widetilde{\mathbf{V}}_{\mathbf{p}}\right]$, each nonlinear vector $\mathbf{C}_{\mathbf{k}}$ or $\mathbf{S}_{\mathbf{k}}$ is reduced to $q$ nonlinear components, the $p=n-q$ other coefficients are equal to zero. Eq. (16) is transformed to separate null components from the others in $\mathbf{W}_{\mathbf{n l}}(\mathbf{X})$ (details are given in [1]):

$$
\left[\begin{array}{ll}
\mathbf{A}_{\mathbf{q q}} & \mathbf{A}_{\mathbf{q p}} \\
\mathbf{A}_{\mathbf{p q}} & \mathbf{A}_{\mathbf{p p}}
\end{array}\right]\left[\begin{array}{l}
\mathbf{X}_{\mathbf{q}} \\
\mathbf{X}_{\mathbf{p}}
\end{array}\right]=\left[\begin{array}{l}
\mathbf{W}_{\text {excit }, \mathbf{q}} \\
\mathbf{W}_{\text {excit }, \mathbf{p}}
\end{array}\right]+\left[\begin{array}{c}
\mathbf{W}_{\mathbf{n l}}\left(\mathbf{X}_{\mathbf{q}}\right) \\
0
\end{array}\right]
$$

The expression serves to write a linear relation between $\mathbf{X}_{\mathbf{p}}$ and $\mathbf{X}_{\mathbf{q}}$ :

$$
\mathbf{X}_{\mathbf{p}}=\mathbf{A}_{\mathbf{p p}}{ }^{-1}\left(\mathbf{W}_{\text {excit }, \mathbf{p}}-\mathbf{A}_{\mathbf{p q}} \mathbf{X}_{\mathbf{q}}\right)
$$

Then Eq. (20) is expressed as an equation in $\mathbf{X}_{\mathbf{q}}$ :

$$
\left(\mathbf{A}_{\mathbf{q q}}-\mathbf{A}_{\mathbf{q p}} \mathbf{A}_{\mathbf{p p}}{ }^{-1} \mathbf{A}_{\mathbf{p q}}\right) \mathbf{X}_{\mathbf{q}}=\mathbf{W}_{\text {excit }, \mathbf{q}}-\mathbf{A}_{\mathbf{q p}} \mathbf{A}_{\mathbf{p p}}{ }^{-1} \mathbf{W}_{\text {excit }, \mathbf{p}}+\mathbf{W}_{\mathbf{n l}}\left(\mathbf{X}_{\mathbf{q}}\right)
$$

This last expression defines the nonlinear optimization problem to be solved:

$$
\mathbf{H}\left(\mathbf{X}_{\mathbf{q}}, \Omega\right)=\left(\mathbf{A}_{\mathbf{q q}}-\mathbf{A}_{\mathbf{q p}} \mathbf{A}_{\mathbf{p p}}{ }^{-1} \mathbf{A}_{\mathbf{p q}}\right) \mathbf{X}_{\mathbf{q}}-\mathbf{W}_{\text {excit }, \mathbf{q}}+\mathbf{A}_{\mathbf{q p}} \mathbf{A}_{\mathbf{p p}}{ }^{-1} \mathbf{W}_{\text {excit }, \mathbf{p}}-\mathbf{W}_{\mathbf{n l}}\left(\mathbf{X}_{\mathbf{q}}\right)=\mathbf{0}
$$

The size of the nonlinear problem to be solved is reduced from $(2 N+1) n$ to $(2 N+1) q$. For this application, with $N=15$, the size is reduced from 3999 to 186 DOFs. The problem (23) is treated using a continuation method. The pseudo-arclength continuation algorithm with a polynomial prediction is implemented. For each solution point $\left(\Omega, \mathbf{X}_{\mathbf{q}}\right)$ computed, the full solution $\left[\mathbf{X}_{\mathbf{q}}, \mathbf{X}_{\mathbf{p}}\right]$ is then calculated using Eq. (21) and translated into the initial basis using the inverse transfer matrix.

\subsection{Test-simulation comparison}

Firstly, the harmonic responses are studied. At the end of the linear modeling process (see Section 3.1), all the parameter values are defined. The nonlinear modeling of the joints introduces then three new parameters: the Coulomb thresholds $F_{\text {Coulomb } \theta y}, F_{\text {Coulomb } x}$ and $F_{\text {Coulomb } z}$ (see Sect 3.3). The values 


\begin{tabular}{lll}
\hline & Parameters set 1 & Parameters set 2 \\
\hline & Experiments with & Experiments with \\
& $0.2<F_{\text {excit }}<10 \mathrm{~m} \cdot \mathrm{s}^{-2}$ & $15<F_{\text {excit }}<30 \mathrm{~m} . \mathrm{s}^{-2}$ \\
\hline$F_{\text {Coulomb } \theta y}$ & $300 \mathrm{~N}$ & $230 \mathrm{~N}$ \\
$F_{\text {Coulomb }}$ & $+\infty$ & $130 \mathrm{~N}$ \\
$F_{\text {Coulomb } z}$ & $+\infty$ & $500 \mathrm{~N}$ \\
\hline
\end{tabular}

Table 3: Updated Coulomb threshold values.

of these parameters remain to be updated. These parameters were optimized so that the numerical FRFs (Frequency Response Functions) best fit the experimental FRFs. At the lowest excitation level, laser vibrometry measurements have proved that only rotational friction occurs. Thus, $F_{C o u l o m b \theta y}$ is updated. Its value is given in Table 3. Below a $10 \mathrm{~m} \mathrm{~s}^{-2}$ excitation level, the other two parameters cannot be estimated. A comparison between the experimental data and the first numerical results obtained is presented in the uper plot of Fig. 17. Above a $10 \mathrm{~m} \mathrm{~s}^{-2}$ excitation level, using the updated value of $F_{\text {Coulomb } \theta y}$ leads to poor agreement between the tests and the simulations, and adding horizontal or vertical friction does not help. The repeatability issue pointed out in Section 2.3 can explain this modeling difficulty. As the blades experience friction wear, the normal contact force can be reduced which implies a reduction of the Coulomb threshold. Considering this evolution, a second value of $F_{C o u l o m b \theta y}$ (lower than the first one) was used for the simulation of the response to excitation levels above $15 \mathrm{~m} \mathrm{~s}^{-2}$. The other two parameters are then updated. The parameter values are presented in Table 3. The lower plot in Fig. 17 presents a comparison between experimental and numerical responses to excitation levels above $15 \mathrm{~m} \mathrm{~s}^{-2}$.

Using these two sets of parameters, numerical results are in good agreement with the experimental results. The main effects due to friction (increase of dissipation and the softening effect) are well reproduced. These results illustrate the relevance of nonlinear modeling and nonlinear vibration simulation methods to simulate the vibration response of assemblies with friction joints.

\subsection{Modal interactions}

The three modal interactions that were measured experimentally are now studied numerically:

- the 1:1 interaction between the transversal responses in the $\mathrm{x}$ and $\mathrm{y}$ directions,

- the 1:9 and 1:11 weak interactions with a $1277 \mathrm{~Hz}$ resonant mode,

- the 1:13 interaction between the first transversal mode and a $1750 \mathrm{~Hz}$ blade pumping mode.

Fig. 18 compares the simulated harmonic responses in the $\mathrm{x}$ and y directions to a $10 \mathrm{~m} \mathrm{~s}^{-2}$ excitation level in the $\mathrm{x}$ direction. The simulated response in the $\mathrm{y}$ direction appears to be discontinuous when friction appears (around $105 \mathrm{~Hz}$ ) and disappears (around $160 \mathrm{~Hz}$ ). The amplitude of this orthogonal 

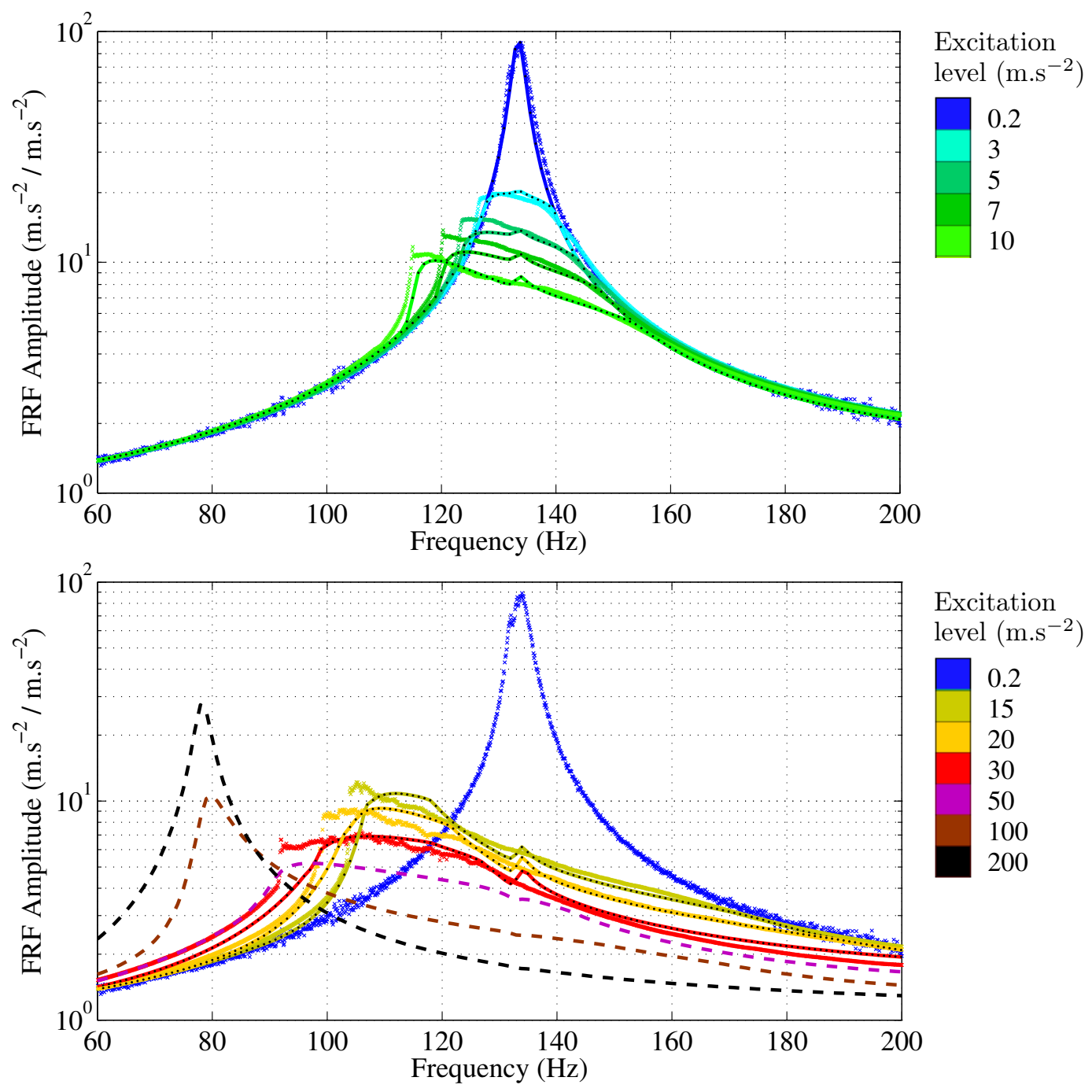

Figure 17: Comparison between experimental (crosses) and simulated (plain curves with black dots) FRF at the top of the central body (point A1 in Fig. 1). The dashed curves represent simulation responses at higher excitation levels than those tested experimentally. 
response is increased $10^{4}$ times in this frequency range. A resonance peak is then observed at $134 \mathrm{~Hz}$ which is the linear resonance frequency of the first transversal twin mode. These results evidence a modal interaction between the first transversal mode and its twin orthogonal mode, in the simulation result. These results agree with the experimental results presented in Fig. 5. The main difference is that the resonance peak observed in the numerical results is less visible in the experimental results. The two twin modes are presented in Fig. 22a and b.

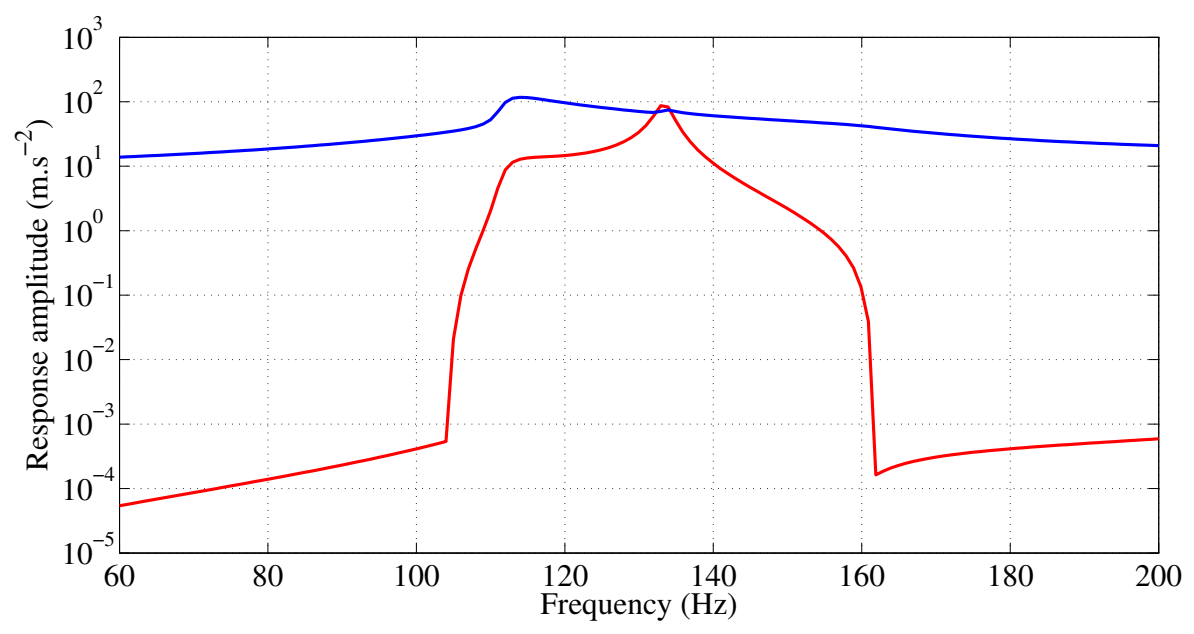

Figure 18: Simulated frequency response at the top of the central body (point A1 in Fig. 1) at a $10 \mathrm{~m} \mathrm{~s}^{-2}$ excitation level in the $\mathrm{x}$ direction. The blue curve corresponds to the response in the $\mathrm{x}$ direction, while the red curve corresponds to the y direction.

Fig. 19 presents the harmonic decomposition of the simulated response, at the top of the central body (point A1 in Fig. 1), at a $10 \mathrm{~m} \mathrm{~s}^{-2}$ excitation level. These harmonic acceleration components $\mathbf{H}^{\mathbf{k}}$ are directly computed from the Fourier coefficients $\mathbf{A}_{\mathbf{k}}$ and $\mathbf{B}_{\mathbf{k}}$ computed in the Harmonic Balance Method:

$$
\left.\mathbf{H}^{\mathbf{k}}\right|_{i}=\sqrt{\left(\left.\mathbf{A}_{\mathbf{k}}\right|_{i}\right)^{2}+\left(\left.\mathbf{B}_{\mathbf{k}}\right|_{i}\right)^{2}} \times(k \Omega)^{2}
$$

where $i$ denotes the index of the DOF.

The higher order harmonic components of the simulated response are at least two orders of magnitude lower than the main harmonic response which is in agreement with experimental results plotted in Fig. 6. As far as modal interactions are concerned, three resonance phenomena can be observed:

- around $143 \mathrm{~Hz}$, for the 9th harmonic (frequency around $143 \times 9=1287 \mathrm{~Hz}$ )

- around $116 \mathrm{~Hz}$, for the 11th harmonic (frequency around $116 \times 11=1276 \mathrm{~Hz}$ )

- around $153 \mathrm{~Hz}$, for the 11th harmonic (frequency around $153 \times 11=1683 \mathrm{~Hz}$ )

The first two resonance phenomena must correspond to modal interactions with the same mode the frequency of which must be around $1280 \mathrm{~Hz}$. In the vicinity of this frequency there is the third 
transversal mode the numerical frequency of which is $1212 \mathrm{~Hz}$ and the first torsion mode the frequency of which is $1293 \mathrm{~Hz}$. As $1287 \mathrm{~Hz}$ and $1276 \mathrm{~Hz}$ are closer to $1293 \mathrm{~Hz}$ than $1212 \mathrm{~Hz}$, the numerical internal resonances pointed out must be between the first transversal mode and the first torsion mode. This mode shape is presented in Fig. 22c. These numerical results provide a new explanation for the experimental 1:9 and 1:11 modal interactions presented in Section 2.4. The frequencies of the two internal resonances are $116 \times 11=1276 \mathrm{~Hz}$ and $142 \times 9=1278 \mathrm{~Hz}$. The resonance frequency of the third transversal mode has been identified at $1240 \mathrm{~Hz}$, from low excitation level measurements. The first torsion mode could not be identified because it is not excited by a horizontal base motion. The experimental modal interaction could then be an interaction with the first torsion mode whose frequency could be around $1277 \mathrm{~Hz}$. Numerical simulations here not only reproduce the experimental results but help in understanding them.

For the last resonance peak pointed out in the 11th harmonic response in Fig. 19, the frequency of the signal is $11 \times 153=1683 \mathrm{~Hz}$. A numerical mode is indeed situated at $1683 \mathrm{~Hz}$. Considering the frequency response in Fig. 15, this can be referred to as the fourth transversal mode of the structure. This numerical resonance at the 11th harmonic is thus a modal interaction between the first and the fourth transversal mode.

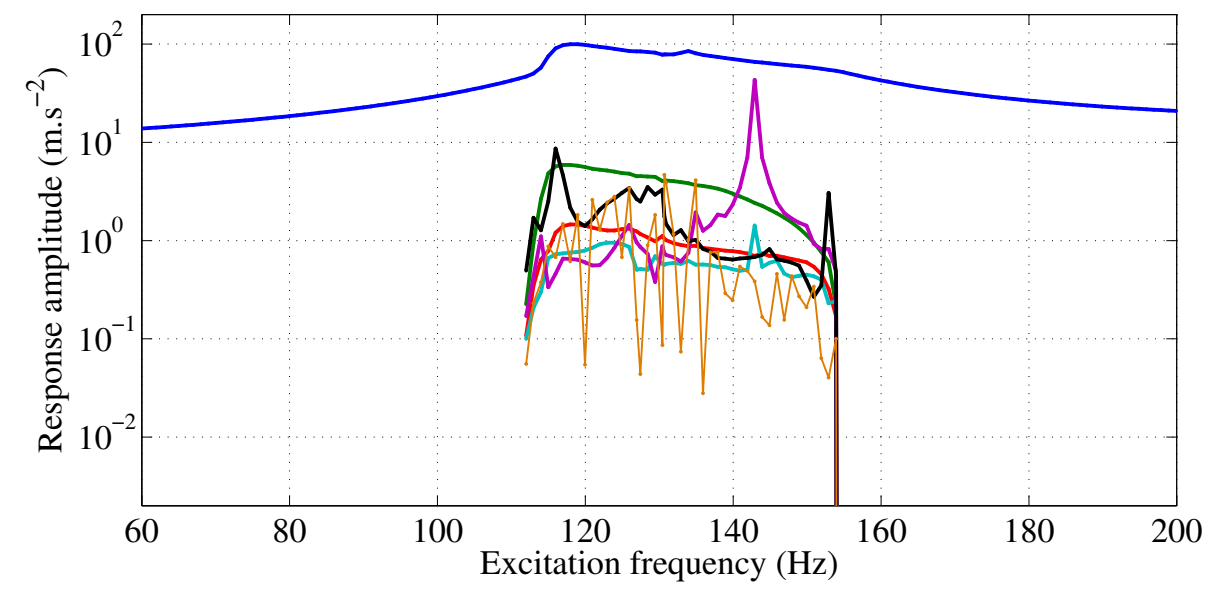

Figure 19: Multi-harmonic simulated response at the top of the central body (point A1 in Fig. 1) at a $10 \mathrm{~m} \mathrm{~s}^{-2}$ excitation level. The odd harmonics are displayed: 1 (blue), 3 (green), 5 (red), 7 (azure), 9 (violet), 11 (black), 13 (orange).

To study the 1:13 modal interaction between the first transversal mode and a blade pumping mode, the movement of the upper edge of the blade has to be computed. The method implemented for that purpose is that presented in [1]. This movement corresponds to that of the reference node "Ref_top_blade" (see Fig. 14) the DOFs of which have deliberately been excluded from the reduction process. The movement of this node is then assumed to be the movement of the slipping point $\mathrm{P}$ in the elastic Coulomb models (see Fig. 16a). For every friction model, the velocity of point $\mathrm{P}$ can 
be easily computed at the same time as the nonlinear force. Its value depends on the state of the Coulomb slider (stick or slip):

$$
v_{P}= \begin{cases}v " R e f \text { contact_zone" } & \text { if stuck } \\ v " R e f_{\text {_contact_zone" }}+\dot{u}_{\text {joint }} & \text { if slipping }\end{cases}
$$

where $u_{\text {joint }}$ is defined by Eq. (7).

Fig. 20 compares the simulated rotation velocity of point $\mathrm{P}$ to that of the external envelope. An alternation of stuck and slipping states is observed. During slipping periods, oscillations in the velocity of the blade occur. Simulation thus reproduces the oscillatory phenomenon observed by the 3D scanning laser vibrometer (see Fig. 10). This phenomenon occurs over a very narrow frequency range. Fig. 21 presents the movement in the orthogonal direction y of a node situated at the center of the blade. The upper plot presents the results at $132 \mathrm{~Hz}$ and the lower plots the results at $135 \mathrm{~Hz}$. The 13th harmonic signal is obvious at $135 \mathrm{~Hz}$ and vanishes at $132 \mathrm{~Hz}$. This is in agreement with the experimental results (see Fig. 13) and is further proof of modal interaction. The 13th harmonic resonance at $135 \mathrm{~Hz}$ corresponds to a signal at $135 \times 13=1755 \mathrm{~Hz}$. The numerical model of the assembly has two twin resonance modes at $1750 \mathrm{~Hz}$. The shape of one of these twin modes is depicted in Fig. 22d. This mode involves mainly blades, and its deformed shape is in agreement with vibrometry measurements at the 13th harmonic depicted in Fig. 12. This numerical result confirms the hypothesis of a modal interaction between the first transversal mode and a blade pumping mode at $1750 \mathrm{~Hz}$.

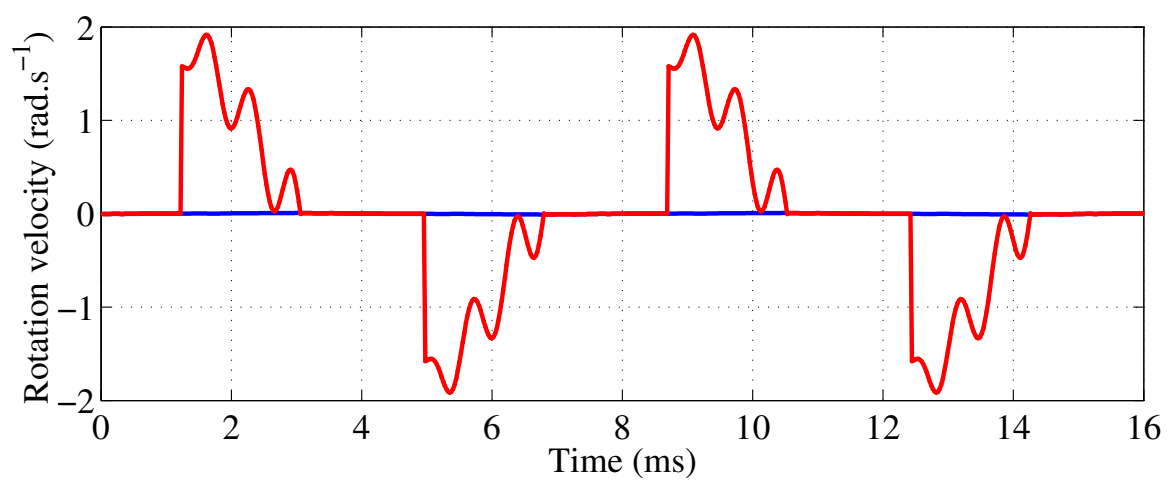

Figure 20: Simulated rotation velocities of the contact zone on the external envelope (blue) and the upper edge of the blade (red). Results are obtained with a $10 \mathrm{~m} \mathrm{~s}^{-2}$ amplitude, $135 \mathrm{~Hz}$ sinusoidal excitation.

\section{Conclusion}

The transversal frequency response of the assembly "Harmony" has been studied both experimentally and numerically. The finite element modeling of the structure, considered in its experimental set-up, 

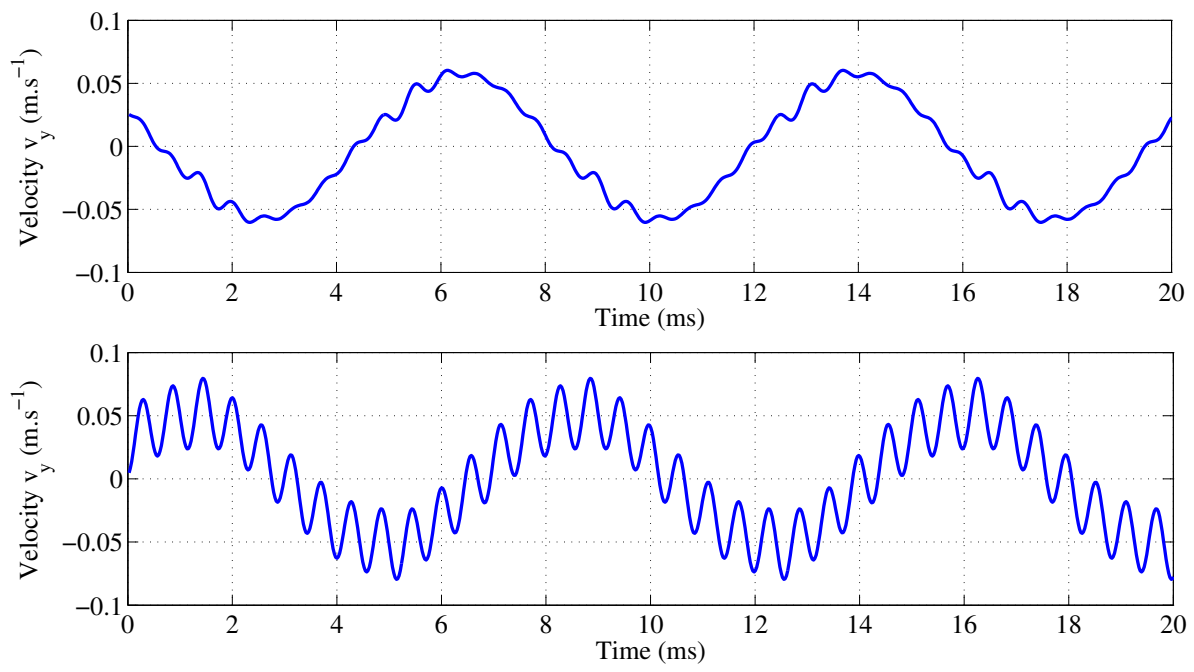

Figure 21: Simulated response in the y direction of a node located at the center of a blade, to a $10 \mathrm{~m} \mathrm{~s}^{-2}$ amplitude sinusoidal excitation at $132 \mathrm{~Hz}$ (upper plot) and $135 \mathrm{~Hz}$ (lower plot).

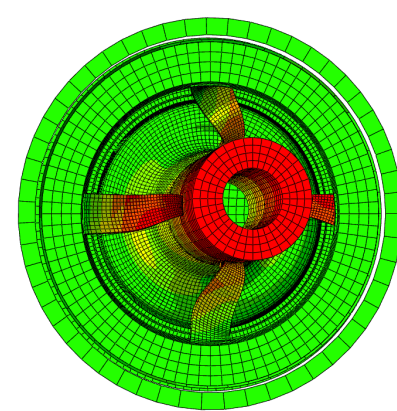

(a) $133.4 \mathrm{~Hz}$

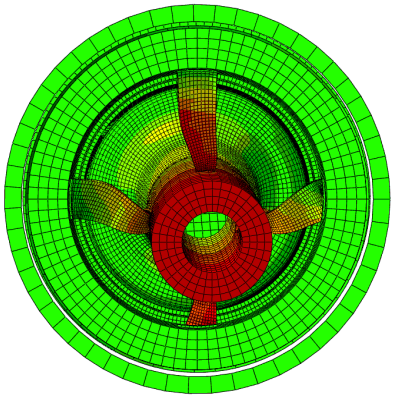

(b) $133.4 \mathrm{~Hz}$

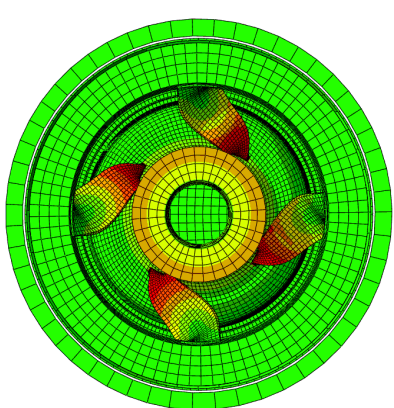

(c) $1293 \mathrm{~Hz}$

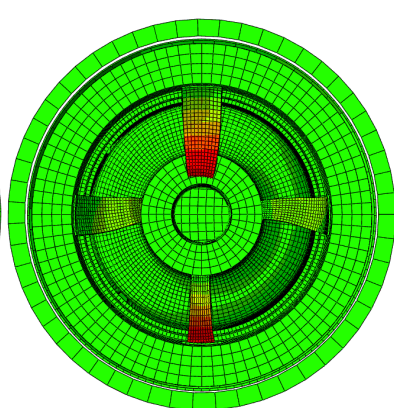

(d) $1750 \mathrm{~Hz}$

Figure 22: Deformed shapes of the numerical modes involved in the modal interactions: first transversal mode along the $\mathrm{x}$ axis (a), first transversal mode along the y axis (b), first torsion mode (c) and blade mode (d) 
is updated to be fully in agreement with the experimental frequency response of the assembly at a low excitation level over the entire range [0-2000] Hz. At a higher excitation level, experimental responses evidence friction with an increase of dissipation and a softening effect. These phenomena are simulated introducing a few friction joint models. The simulation methodology proposed uses well-known structural dynamics methods: model reduction using a substructuring method, the Harmonic Balance Method coupled with a condensation algorithm and a continuation method. This simulation process makes it possible to compute the nonlinear frequency responses of the model. The increase of dissipation and the softening effects are very well reproduced when two sets of parameters are considered. This evolution of the model parameters is justified by the experimental evidence of fretting wear of the contact joints. At this step, the numerical methodology has proved to be able to treat friction induced by transversal excitations as well as friction induced by axial excitations. The transversal case is more complex in the sense that vertical, horizontal and rotational slipping movements can occur at the same time in the joints. This study is thus first a new validation that the numerical methodology proposed is able to simulate the vibration response of assemblies with friction joints. The updating step in the modeling process highlighted considerable deviations from one experiment to another. These deviations are due both to aleatoric uncertainties and to abrasion. In order to improve the modeling, a stochastic model should be considered. The introduction of stochastic parameters in a dynamic problem formulated using the Harmonic Balance Method has been studied by Didier [25, 26].

The main contribution of the present paper is the study of modal interactions. Three modal interactions have been identified in the experimental response of the test structure: the main transversal mode interacts with its twin orthogonal mode (1:1 modal interaction), with a structure mode at 1277 $\mathrm{Hz}$ (1:9 and 1:11 weak modal interaction) and with a blade pumping mode at $1750 \mathrm{~Hz}$. This last interaction was identified thanks to scanning laser vibrometry measurements. Simulation results present exactly the same evidence of modal interactions for the three of them. Moreover, considering the 1:9 and 1:11 modal interactions, numerical results show an internal resonance between the first transversal mode and the torsion mode. In this case, numerical simulations both reproduce the experimental results and help to understand them. This paper thus shows that modal interactions can occur in the vibration response of assemblies with friction joints. Moreover, the numerical results presented prove the efficiency of the proposed numerical methodology to predict complex dynamic forms of behavior.

\section{Acknowledgements}

The authors gratefully acknowledge the support of the CEA/CESTA teams who helped with this study. They acknowledge in particular the experimental service which provided the measurements presented in this paper.

J-J. Sinou acknowledges the support of the Institut Universitaire de France. 


\section{References}

[1] M. Claeys, J.-J. Sinou, J.-P. Lambelin, and R. Todeschini. Experiments and numerical simulations of nonlinear vibration responses of an assembly with friction joints-application on a test structure named "harmony". Mechanical Systems and Signal Processing, 70:1097-1116, 2016.

[2] M. Kim, V. Belsky, and M. Belyi. Substructure generation using automated multilevel substructuring, 2013. Patent US 2013/0124150A1 and EP 2597578A1.

[3] E. Sarrouy and J-J. Sinou. Non-linear periodic and quasi-periodic vibrations in mechanical systems - on the use of the harmonic balance methods. In Farzad Ebrahimi, editor, Advances in Vibration Analysis Research, Chapter 21. INTECH, Open Access Publisher, 2011.

[4] J.-J. Sinou. Non-linear dynamics and contacts of an unbalanced flexible rotor supported on ball bearings. Mechanism and Machine Theory, 44:1713-1732, 2009.

[5] A.H. Nayfeh and D.T. Mook. Nonlinear Oscillations. John Wiley \& Sons, New York, 1979.

[6] A.H. Nayfeh. Nonlinear Interactions. John Wiley \& Sons, New York, 2000.

[7] A.G. Haddow, A.D.S. Barr, and D.T. Mook. Theoretical and experimental study of modal interaction in two-degree-of-freedom structure. Journal of Sound and Vibration, 97(3):451-473, 1984.

[8] A.H. Nayfeh. On the undesirable roll characteristics of ships in regular seas. Journal of Ship Research, 32(2):92-100, 1988.

[9] O. Thomas, C. Touzé, and A. Chaigne. Non-linear vibrations of free-edge thin spherical shells: modal interaction rules and 1:1:2 internal resonance. International Journal of Solids and Structures, 42:3339-3373, 2005.

[10] M. Monteil, C. Touzé, O. Thomas, and S. Benacchio. Nonlinear forced vibrations of thin structures with tuned eigenfrequencies: the cases of 1:2:4 and 1:2:2 internal resonances. Nonlinear Dynamics, $75: 175-200,2014$.

[11] M. Monteil, O. Thomas, and C. Touzé. Identification of mode couplings in nonlinear vibrations of the steelpan. Applied Acoustics, 89:1-15, 2015.

[12] J.-P. Noel, L. Renson, and G. Kerschen. Complex dynamics of a nonlinear aerospace structure: experimental identification and modal interactions. Journal of Sound and Vibration, 333:2588$2607,2014$.

[13] L. Renson, J.-P. Noel, and G. Kerschen. Complex dynamics of a nonlinear aerospace structure: numerical continuation and normal modes. Nonlinear Dynamics, 79:1293-1309, 2015. 
[14] M. Claeys, J.-J. Sinou, J.-P. Lambelin, and B. Alcoverro. Multi-harmonic measurements and numerical simulations of nonlinear vibrations of a beam with non-ideal boundary conditions. Communications in Nonlinear Science and Numerical Simulation, 19:4196-4212, 2014.

[15] Polytec. Psv-500-3d scanning vibrometer datasheet. URL http://www.polytec.com.

[16] R.R. Craig and M.C.C. Bampton. Coupling of substructure for dynamic analysis. AIAA Journal, 1(2):1313-1319, 1968.

[17] J. Guillen and C. Pierre. An efficient, hybrid, frequency-time domain method for the dynamics of large-scale dry-friction damped structural systems. In IUTAM Symposium on Unilateral Multibody Contacts, pages 169-178. Springer, 1999.

[18] H. Festjens, G. Chevallier, and J.-L. Dion. Nonlinear model order reduction of jointed structures for dynamical analysis. Journal of Sound and Vibration, 333(7):2100-2113, 2014.

[19] P.R. Dahl. Solid friction damping of mechanical vibrations. AIAA Journal of Guidance, Control and Dynamics, 14:1675-1682, 1976.

[20] W.D. Iwan. A distributed-element model for hysteresis and its steady-state dynamic response. Journal of Applied Mechanics, 33:893-900, 1966.

[21] L. Gaul and J. Lenz. Nonlinear dynamics of structures assembled by bolted joints. Acta Mechanica, 125(1-4):169-181, 1997.

[22] L. Gaul and R. Nitsche. The role of friction in mechanical joints. Applied Mechanics Reviews, 54(2):93-106, 2001.

[23] T.M. Cameron and J.H. Griffin. An alternating frequency time domain method for calculating the steady state response of nonlinear dynamic systems. ASME Journal of Applied Mechanics, 56:149-154, 1989.

[24] E.J. Hahn and P.Y. Chen. Harmonic balance analysis of general squeeze film damped multidegreeof-freedom rotor bearing systems. Journal of Tribology, 116:499-507, 1994.

[25] J. Didier, J.-J. Sinou, and B. Faverjon. Study of the non-linear dynamic response of a rotor system with faults and uncertainties. Journal of Sound and Vibration, 331:671-703, 2012.

[26] J. Didier, J.-J. Sinou, and B. Faverjon. Nonlinear vibrations of a mechanical system with nonregular nonlinearities and uncertainties. Communications in Nonlinear Science and Numerical Simulation, 18:3250-3270, 2013. 